\title{
Resonances, radiation damping and instability in Hamiltonian nonlinear wave equations
}

\author{
A. Soffer ${ }^{1}$, M.I. Weinstein ${ }^{2, *}$ \\ ${ }^{1}$ Department of Mathematics, Rutgers University, New Brunswick, NJ, USA \\ ${ }^{2}$ Department of Mathematics, University of Michigan, Ann Arbor, MI USA
}

Oblatum: 6-XI-1998 \& 12-VI-1998 / Published online: 14 January 1999

\begin{abstract}
We consider a class of nonlinear Klein-Gordon equations which are Hamiltonian and are perturbations of linear dispersive equations. The unperturbed dynamical system has a bound state, a spatially localized and time periodic solution. We show that, for generic nonlinear Hamiltonian perturbations, all small amplitude solutions decay to zero as time tends to infinity at an anomalously slow rate. In particular, spatially localized and time-periodic solutions of the linear problem are destroyed by generic nonlinear Hamiltonian perturbations via slow radiation of energy to infinity. These solutions can therefore be thought of as metastable states. The main mechanism is a nonlinear resonant interaction of bound states (eigenfunctions) and radiation (continuous spectral modes), leading to energy transfer from the discrete to continuum modes. This is in contrast to the KAM theory in which appropriate nonresonance conditions imply the persistence of invariant tori. A hypothesis ensuring that such a resonance takes place is a nonlinear analogue of the Fermi golden rule, arising in the theory of resonances in quantum mechanics. The techniques used involve: (i) a time-dependent method developed by the authors for the treatment of the quantum resonance problem and perturbations of embedded eigenvalues, (ii) a generalization of the Hamiltonian normal form appropriate for infinite dimensional dispersive systems and (iii) ideas from scattering theory. The arguments are quite general and we expect them to apply to a large class of
\end{abstract}

\footnotetext{
* Present address

Mathematical Sciences Research Bell Labs 2C-358

Lucent Technologies, Murray Hill, NJ, USA
} 
systems which can be viewed as the interaction of finite dimensional and infinite dimensional dispersive dynamical systems, or as a system of particles coupled to a field.

\section{Contents}

1. Introduction and statement of main results - Smile of the Cheshire Cat

2. Linear analysis

3. Existence theory

4. Isolation of the key resonant terms and formulation as a coupled finite and infinite dimensional dynamical system

5. Dispersive Hamiltonian normal form

6. Asymptotic behavior of solutions of perturbed normal form equations

7. Asymptotic behavior of solutions of the nonlinear Klein Gordon equation

8. Summary and discussion

\section{Introduction and statement of main results - Smile of the Cheshire Cat*}

Structural stability and the persistence of coherent structures under perturbations of a dynamical system are fundamental issues in dynamical systems theory with implications in many fields of application. In the context of discrete, finite dimensional Hamiltonian systems, this issue is addressed by the celebrated KAM theorem [3], which guarantees the persistence of most invariant tori of the unperturbed dynamics under small Hamiltonian perturbations. For infinite dimensional systems, defined by Hamiltonian partial differential equations (PDEs), KAM type methods have recently been used to obtain results on the persistence of periodic and quasi-periodic solutions, in the case where solutions are defined on compact spatial domains with appropriate boundary conditions [4], [17], [37]. Variational methods have also been used to study this problem; see, for example, [6]. The compactness of the spatial domain ensures discreteness of the spectrum associated with the unperturbed dynamics. Therefore this situation is the generalization of the finite dimensional case to systems with an infinite number of discrete oscillators and frequencies.

In this paper we consider these questions in the context of Hamiltonian systems for which the unperturbed dynamics has associated with it discrete and continuous spectrum. This situation arises in the study of Hamiltonian PDEs governing functions defined on unbounded spatial domains or, more generally, extended systems. The physical picture is that of a system which can be viewed as an 
interaction between one or more discrete oscillators and a field or continuous medium. In contrast to the KAM theory, where nonresonance implies persistence, we find here that resonant nonlinear interaction between discrete (bound state) modes and continuum (dispersive radiation) modes leads to energy transfer from the discrete to continuum modes. This mechanism is responsible for the eventual time-decay and nonpersistence of trapped states. The rate of timedecay, however, is very slow and hence such a trapped state can be thought of as a metastable state.

The methods we develop are applicable to a large class of problems which can be viewed schematically in terms of "particle-field interactions". In the present work, we have not attempted to present general results under weak hypotheses but rather have endeavored to illustrate, by way of example, this widely occurring phenomenon and clearly present the strategy for its analysis. A more general point of view will be taken up in future work. The approach we use was motivated and in part developed in the context of our study of a class of nonlinear Schrödinger equations with multiple nonlinear bound states and the quantum resonance problem [60], [61], [62], [63]. See also [11], [12] and [46]. Related problems are also considered in [32] and [44].

We begin with a linear dispersive Hamiltonian PDE for a function $u(x, t), x \in \mathbb{R}^{n}$ and $t>0$. Suppose that this system has spatially localized and time-periodic solutions. Such solutions are often called bound states. A typical solution to such a linear system consists of (i) a non-decaying part, expressible as a linear combination of bound states, plus (ii) a part which decays to zero in suitable norms (dispersion). This paper is devoted to the study of the following questions:

(1) Do small amplitude spatially localized and time-periodic solutions persist for typical non-linear and Hamiltonian perturbations?

(2) What is the character of general small amplitude solutions to the perturbed dynamics?

(3) How are the structures of the unperturbed dynamics manifested in the perturbed dynamics?

Representative of the class of equations of interest is the nonlinear Klein-Gordon equation:

$$
\partial_{t}^{2} u=\left(\Delta-V(x)-m^{2}\right) u+\lambda f(u), \quad \lambda \in \mathbb{R}
$$

with $f(u)$ real-valued, smooth in a neighborhood of $u=0$ and having an expansion:

$$
f(u)=u^{3}+\mathcal{O}\left(u^{4}\right) .
$$


Here, $u:(x, t) \mapsto u(x, t) \in \mathbb{R}, x \in \mathbb{R}^{3}$, and $t>0$. We shall restrict our large time asymptotic analysis to the case $f(u)=u^{3}$. The more general case (1.2) can be treated by the technique of this paper by making suitable modification of $W^{s, p}$ norms used.

We consider (1.1) with Cauchy data:

$$
u(x, 0)=u_{0}(x), \text { and } \partial_{t} u(x, 0)=u_{1}(x) .
$$

Equation (1.1) is a Hamiltonian system with energy:

$$
\mathscr{E}\left[u, \partial_{t} u\right] \equiv \frac{1}{2} \int\left(\partial_{t} u\right)^{2}+|\nabla u|^{2}+m^{2} u^{2}+V(x) u^{2} d x+\lambda \int F(u) d x
$$

where $F^{\prime}(u)=f(u)$ and $F(0)=0$.

In the context of equations of type (1.1), we have found the following answers to questions (1), (2) and (3).

(A1) In a small open neighborhood of the origin, there are no periodic or quasiperiodic solutions; Corollary 1.1.

(A2) All solutions in this neighborhood tend to zero (radiate) as $t \rightarrow \infty$; Theorem 1.1.

(A3) The time decay of solutions is anomalously slow*, i.e. a rate which is slower than the free dispersive rate; Theorem 1.1.

Dynamical systems of the type we analyze appear in a number of physical settings. Consider a nonlinear medium in which waves can propagate. If the medium has local inhomogeneities, defects or impurities, these arise in the mathematical model as a spatially dependent coefficient in the equation (e.g. localized potential). Such perturbations of the original homogeneous (translation invariant) dynamics introduce new modes into the system (impurity modes) which can trap some of the energy and affect the time evolution of the system; see [41], [35], [73].

Let $\langle K\rangle=\left(1+|K|^{2}\right)^{\frac{1}{2}}$. For the nonlinear Klein-Gordon equation, (1.1), we prove the following result.

Theorem 1.1. Let $V(x)$ be real-valued and such that

(V1) For $\delta>5$ and $|\alpha| \leq 2,\left|\partial^{\alpha} V(x)\right| \leq C_{\alpha}\langle x\rangle^{-\delta}$.

(V2) $(-\Delta+1)^{-1}\left((x \cdot \nabla)^{l} V(x)\right)(-\Delta+1)^{-1}$ is bounded on $L^{2}$ for $|l| \leq N_{*}$ with $N_{*} \geq 10$.

(V3) Zero is not a resonance of the operator $-\Delta+V$; see [31], [72]. Assume the operator

$$
B^{2}=-\Delta+V(x)+m^{2}
$$


has continuous spectrum, $\sigma_{\mathrm{cont}}(H)=\left[\mathrm{m}^{2}, \infty\right)$, and a unique strictly positive simple eigenvalue, $\Omega^{2}<m^{2}$ with associated normalized eigenfunction, $\varphi$ :

$$
B^{2} \varphi=\Omega^{2} \varphi .
$$

Correspondingly, the linear Klein-Gordon equation (1.1), with $\lambda=0$, has a two-parameter family of spatially localized and time-periodic solutions of the form:

$$
u(x, t)=R \cos (\Omega t+\theta) \varphi(x)
$$

Assume the resonance condition

$$
\Gamma \equiv \frac{\pi}{3 \Omega}\left(\mathbf{P}_{\mathrm{c}} \varphi^{3}, \delta(B-3 \Omega) \mathbf{P}_{\mathrm{c}} \varphi^{3}\right) \equiv \frac{\pi}{3 \Omega}\left|\left(\mathscr{F}_{\mathrm{c}} \varphi^{3}\right)(3 \Omega)\right|^{2}>0 .
$$

Here, $\mathbf{P}_{\mathrm{c}}$ denotes the projection onto the continuous spectral part of $B$ and $\mathscr{F}_{\mathrm{c}}$ denotes the Fourier transform relative to the continuous spectral part of $B$.

Assume that the initial data $u_{0}, u_{1}$ are such that the norm $\left\|u_{0}\right\|_{w^{2,2} \cap w^{2,1}}$ and $\left\|u_{1}\right\|_{w^{1,2} \cap w^{1,1}}$ are sufficiently small. Then, the solution of the initial value problem for (1.1), with $\lambda \neq 0$ and $f(u)=u^{3}$ decays as $t \rightarrow \pm \infty$. The solution $u(x, t)$ has the following expansion as $t \rightarrow \pm \infty$ :

$$
u(x, t)=R(t) \cos (\Omega t+\theta(t)) \varphi(x)+\eta(x, t)
$$

where

$$
R(t)=\mathcal{O}\left(|t|^{-\frac{1}{4}}\right), \theta(t)=\mathcal{O}\left(|t|^{\frac{1}{2}}\right), \text { and }\|\eta(\cdot, t)\|_{8}=\mathcal{O}\left(|t|^{-\frac{3}{4}}\right) .
$$

More precisely,

$$
\begin{aligned}
R & =\tilde{R}+\mathcal{O}\left(|\tilde{R}|^{2}\right),(|\tilde{R}| \text { small }) \\
\tilde{R} & =2^{\frac{1}{4}} \tilde{R}_{0}\left(1+\frac{3}{4} \tilde{R}_{0}^{4} \Omega^{-1} \lambda^{2} \Gamma|t|\right)^{-\frac{1}{4}} \cdot\left(1+\mathcal{O}\left(|t|^{-\delta}\right)\right), \quad \delta>0 \\
R(0) & =R_{0}, R_{0}^{2}=\left|\left(\varphi, u_{0}\right)\right|^{2}+\Omega^{-2}\left|\left(\varphi, u_{1}\right)\right|^{2}
\end{aligned}
$$

Corollary 1.1. Under the hypotheses of Theorem 1.1, there are no periodic or quasiperiodic orbits of the flow $t \mapsto\left(u(t), \partial_{t} u(t)\right)$ defined by 
(1.1) in a sufficiently small neighborhood of the origin in the space $\left(W^{2,1} \cap W^{2,2}\right) \times\left(W^{1,2} \cap W^{1,1}\right)$.

It is interesting to contrast our results with those known for Hamiltonian partial differential equations for a function $u(x, t)$, where $x$ varies over a compact spatial domain, e.g. periodic or Dirichelt boundary conditions [4], [17], [37]. For nonlinear wave equations of the form, (1.1), with periodic boundary conditions in $x$, KAM type results have been proved; invariant tori, associated with a nonresonance condition persist under small perturbations. The nonresonance hypotheses of such results fail in the current context, a consequence of the continuous spectrum associated with unbounded spatial domains. In our situation, non-vanishing resonant coupling (condition (1.8)) provides the mechanism for the radiative decay and therefore nonpersistence of localized periodic solutions.

Remarks. (1) The condition (1.8) is a nonlinear variant of the Fermi golden rule arising in quantum mechanics; see, for example, [18], [62]. This condition holds generically in the space of potentials satisfying the hypothesis of the theorem. Note that the condition (1.8) implies that

$$
3 \Omega \in \sigma_{\text {cont }}(B)
$$

i.e. the frequency, $3 \Omega$, generated by the cubic nonlinearity lies in the continuous spectrum of $B$. The hypothesis (1.8) ensures a nonvanishing coupling to the continuous spectrum.

(2) The regularity and decay hypotheses on $V(x)$ are related to the techniques we use to obtain suitable decay estimates on the linear evolution in $L^{2}\left(\langle x\rangle^{-\delta} d x\right)$ and $L^{p}$; see Section 2.

(3) Persistence of dynamically stable bound states for special perturbations: There are nonlinear perturbations of the linear KleinGordon equation, (1.1) with $\lambda=0$, for which there is a persistence of time-periodic and spatially localized solutions. Suppose we extend our considerations to the class of complex-valued solutions: $u: \mathbb{R}^{n} \times \mathbb{R} \rightarrow \mathbb{C}$, and study the perturbed equation:

$$
\left(\partial_{t}^{2}-\Delta+V(x)+\lambda|u|^{p-1}\right) u=0,
$$

where $1<p<\infty$ for $n=1,2$ and $1<p<\frac{n+2}{n-2}$ for $n \geq 3$. Unlike (1.1), equation (1.12) has the symmetry $u \mapsto u \mathrm{e}^{\mathrm{i} \gamma}$ and it has been shown [51] that (1.12) has time periodic and spatially localized solutions of the form $\mathrm{e}^{\mathrm{i} \omega t} \Phi(x ; \omega)$ with $\Phi \in H^{1}$, which bifurcate from the zero solution at the point eigenvalue of $-\Delta+V(x)-\omega^{2}$, by 
global variational [39], [65] and local bifurcation methods [45]. There are numerous other examples of equations for which the persistence of coherent structures under perturbations is linked to the perturbation respecting a certain symmetry of the unperturbed problem. The stability of small amplitude bifurcating states of (1.12) can be proved using the methods of [24], [70]. An asymptotic stability and scattering theory has been developed for nonlinear Schrödinger dynamics (NLS) in [60]. If the potential $V(x)$ supports more than one bound state, the above methods can be used to show the persistence of nonlinear excited states. However, it is shown in [63] that the NLS excited states are unstable, due to a resonant mechanism of the type studied here for (1.1).

(4) In contrast with the time-decay rates associated with linear dispersive equations, the time-decay rates of typical solutions described by Theorem 1.1 is anomalously slow. See Section 8 for a discussion of this point.

We now present an outline of the ideas behind our analysis. For $\lambda=0$, solutions of equation (1.1) are naturally decomposed into their discrete and continuous spectral components:

$$
u(x, t)=a(t) \varphi(x)+\eta(x, t) \quad(\varphi, \eta(\cdot, t))=0,
$$

where $(f, g)$ denotes the usual complex inner product on $L^{2}$. The functions $a(t)$ and $\eta(x, t)$ satisfy system of decoupled equations:

$$
\begin{aligned}
& a^{\prime \prime}+\Omega^{2} a=0, \\
& \partial_{t}^{2} \eta+B^{2} \eta=0,
\end{aligned}
$$

with initial data

$$
\begin{aligned}
a(0) & =\left(\varphi, u_{0}\right), \quad a^{\prime}(0)=\left(\varphi, u_{1}\right) \\
\eta(x, 0) & =\mathbf{P}_{\mathrm{c}} u_{0}, \quad \partial_{t} \eta(x, 0)=\mathbf{P}_{\mathrm{c}} u_{1}
\end{aligned}
$$

For $\lambda \neq 0$, and for small amplitude initial conditions, we use the same decomposition, (1.13). Now the discrete and the continuum modes are coupled and the dynamics are qualitatively described by the following model system:

$$
\begin{aligned}
& a^{\prime \prime}+\Omega^{2} a=3 \lambda a^{2}(\chi, \eta(\cdot, t)) \\
& \partial_{t}^{2} \eta-\Delta \eta+m^{2} \eta=\lambda a^{3} \chi .
\end{aligned}
$$


Here, $\chi(x)$ is a localized function of $x$; in particular, $\chi=\varphi^{3}$, and we assume (see (1.8)) $3 \Omega>m$. In selecting the model problem (1.171.18), we have replaced $B^{2}$, restricted to its continuous spectral part, by $B_{0}^{2}=-\Delta+m^{2}$, which intuitively should lead to the same qualitative result.

The system (1.17-1.18) can be interpreted as a system governing the dynamics of discrete oscillator, with amplitude $a(t)$ and natural frequency $\Omega$, coupled to a continuous medium in which waves, of amplitude $\eta(x, t)$, propagate, or as an oscillating particle coupled to a field. ${ }^{1}$

The system $(1.17-1.18)$ is a Hamiltonian system with conserved total energy functional:

$$
\begin{aligned}
\tilde{\mathscr{E}}\left[\eta, \partial_{t} \eta, a, a^{\prime}\right] \equiv & \frac{1}{2} \int\left(\partial_{t} \eta\right)^{2}+|\nabla \eta|^{2}+m^{2} \eta^{2} d x+\frac{1}{2}\left(a^{\prime 2}+\Omega^{2} a^{2}\right) \\
& -\lambda a^{3} \int \chi(x) \eta(x, t) d x
\end{aligned}
$$

We now solve (1.18) and substitute the result into (1.17). Note that, to leading order, solutions of (1.17) oscillate with frequency $\Omega$. Therefore the $a^{3}$ term in (1.18) acts as an external driving force with a $3 \Omega$ frequency component. Since $9 \Omega^{2}$ is larger then $m^{2}$, a nonlinear resonance of the oscillator with the continuum takes place. To calculate the effect of this resonance requires a careful analysis involving (i) a study of singular limits of resolvents as an eigenvalue parameter approaches the continuous spectrum (see Section 4) and (ii) and the derivation of a normal form which is natural for an infinite dimensional conservative system with dispersion (see Section 5). This leads to an equation of the following type for $a(t)$ (or rather some nearidentity transform of it):

$$
a^{\prime \prime}+\left(\Omega^{2}+\mathcal{O}\left(|a|^{2}\right)\right) a=-\Gamma r^{4} a^{\prime}, \quad t>0
$$

where $r=\mathcal{O}(|a|)$, and $\Gamma>0$ is the positive number given in (1.8). For the model system (1.18), $\mathscr{F}_{\mathrm{c}}$ in (1.8) is replaced by the usual Fourier transform. This is the equation of a nonlinearly damped oscillator:

$$
\frac{d}{d t}\left(\left(a^{\prime}\right)^{2}+\left[\Omega^{2}+\mathcal{O}\left(|a|^{2}\right)\right] a^{2}\right)=-2 \Gamma r^{4}\left(a^{\prime}\right)^{2}<0, \text { for } t>0
$$

\footnotetext{
${ }^{1}$ A related example is a model introduced in 1900 by H. Lamb [38], governing the oscillations of mass-spring-string system. See also [7], [21], [53], [30].
} 
Therefore, nonlinear resonance is responsible for internal damping in the system; energy is lost or rather transferred from the discrete oscillator into the field or continuous medium, where it is propagated to infinity as dispersive waves. Solutions of (1.20) decay with a rate $\mathcal{O}\left(t^{-1 / 4}\right)$, and it follows that $\|\eta(\cdot, t)\|_{\infty}=\mathcal{O}\left(t^{-3 / 4}\right)$. Note, however that the total energy of the system, an $H^{1}$ type quantity, is conserved. In (1.20), we have neglected higher order effects coming from the continuous spectral part of $H$. These, it turns out, have a small effect and can be treated perturbatively.

Aspects of the analysis are related to our recent treatment of the quantum resonance problem [61], [62]. The situation with quantum resonances can be summarised briefly as follows. Let $H_{0}$ be a selfadjoint operator having an eigenvalue, $\lambda_{0}$, embedded in its continuous spectrum, with corresponding normalized eigenfunction $\psi_{0}$. Let $W$ be a small localized symmetric perturbation, satisfying the (generically valid) Fermi golden rule resonance condition:

$$
\Gamma_{0} \equiv \pi\left(W \psi_{0}, \delta\left(H_{0}-\lambda_{0}\right) \mathbf{P}_{c} W \psi_{0}\right) \neq 0 .
$$

Then in a neighborhood of $\lambda_{0}$, we show that the spectrum of $H=H_{0}+W$ is absolutely continuous by proving that all solutions of the Schrödinger equation:

$$
\mathrm{i} \partial_{t} \psi=H \psi=\left(H_{0}+W\right) \psi,
$$

with initial data which is spectrally localized (with respect to $H$ ) in a neighborhood of $\lambda_{0}$, decay to zero in a local energy norm as $t \rightarrow \pm \infty$.

Such solutions are characterized by exponential time-decay for an initial transient period, and then algebraic (dispersive decay) thereafter. During this transient period, $A(t)$, the projection onto the mode $\psi_{0}$, is governed by the equation:

$$
A^{\prime}(t)=\left(\mathrm{i} \lambda_{*}-\Gamma_{0}\right) A(t), \quad t>0
$$

where $\lambda_{*} \sim \lambda_{0}+\left(\psi_{0}, W \psi_{0}\right)$, and by (1.22), $\Gamma_{0}>0$.

In the class of nonlinear problems under consideration, if we express the amplitude, $a(t)$, as

$$
a(t)=A(t) \mathrm{e}^{\mathrm{i} \Omega t}+\bar{A}(t) \mathrm{e}^{-\mathrm{i} \Omega t} .
$$

Then we find after a near-identity transformation an equation of the form:

$$
A^{\prime}=\mathrm{i} c_{21}|A|^{2} A+\left(\mathrm{i} c_{32}-\frac{3}{4} \frac{\lambda^{2}}{\Omega} \Gamma\right)|A|^{4} A, \quad t>0 .
$$


From this, the $\mathcal{O}\left(t^{-1 / 4}\right)$ behavior is evident. Equations (1.25-1.26) lead to (1.20). As in (1.20), we have in (1.24) and (1.26) neglected the higher order coupling to the continuous spectral (radiation) component of the solution. These contributions are treated perturbatively.

Remarks. (1) Lamb shift: Note that in the nonlinear problem, asymptotically there is no Lamb shift type correction to the frequency; the frequency shift is $\mathcal{O}\left(|A|^{2}\right)=\mathcal{O}\left(t^{-1 / 2}\right)$ as $t \rightarrow \pm \infty$.

(2) Emergence of irreversible behavior from reversible dynamics; dissipation through dispersion: Being Hamiltonian, the underlying equation of motion, (1.1), is time reversible. In particular, the equation has the invariance: $u(x, t) \mapsto u(x,-t)$. Yet, the equation in (1.26) is clearly not time-reversible. This apparent paradox is related to the "E-prescription" discussed in Section 4 and Proposition 2.1; the singular limit

$$
\lim _{\varepsilon \downarrow 0} \exp \left(\mathrm{i} \sqrt{-\Delta+m^{2}} t\right)\left(-\Delta+m^{2}-E \pm \mathrm{i} \varepsilon\right)^{-1}
$$

satisfies a local decay estimate as $t \rightarrow \pm \infty$. For $t<0$, the corresponding equation for $A(t)$ would have $-\Gamma$ replaced by $+\Gamma$; cf. [2], [12], [61], [62].

The nonpersistence of small amplitude spatially localized and time-periodic or quasiperiodic solutions to (1.1) has been studied in [56], [57]. In this work, the phenomenon of nonpersistence is formulated as a question concerning the instability of embedded eigenvalues of a suitable linear self-adjoint operator. A result on structural instability is proved; under the hypothesis (1.8), timeperiodic or quasiperiodic solutions of the linear problem equation $(\lambda=0)$ do not continue to nearby solutions of the nonlinear problem $(\lambda \neq 0)$.

The question of nonpersistence of periodic solutions has also been considered extensively in the context of the sine-Gordon equation

$$
\partial_{t}^{2} u=\partial_{x}^{2} u-\sin u
$$

Spatially localized and time-periodic solutions of the sine-Gordon equation are called breathers and the question of their persistence under small Hamiltonian perturbations in the dynamics has been the subject of extensive investigations. See, for example, [54], [16], [13] [69], [8], [9], [19], [34], [47], [66], [42]. 
Analytical, formal asymptotic and numerical studies strongly suggest that for typical Hamiltonian perturbations of the sineGordon equation, for example, the $\phi^{4}$ model:

$$
\partial_{t}^{2} u=\partial_{x}^{2} u-u+u^{3}
$$

no small amplitude breathers exist and that solutions obtained from spatially localized initial data radiate to zero very slowly as $t$ tends to infinity. ${ }^{2}$ We believe this is related to the mechanism for slow radiative decay, as explained by Theorem 1.1 in the context of (1.1).

Finally, we wish to comment on the connection between our work and the approach taken in [56], [8], [19], and [34]. As in the continuation theory of periodic solutions of ordinary differential equations [15], it is natural to seek a periodic solution of (1.1) for $\lambda \neq 0$ which behaves, as the amplitude $a$ tends to zero, like a solution (1.7) of the linear limit problem. The equation is autonomous with respect to time, so we seek a $2 \pi \Omega_{a}^{-1}$-periodic solution for $\lambda \neq 0$ with an amplitude dependent period. Since we do not know the period á priori, it is convenient to define

$$
u(x, t)=U_{a}(x, s), \quad s=\Omega_{a} t
$$

and require that $U_{a}(x, s)$ be $2 \pi$ periodic in $s$. Thus (1.1) becomes

$$
\left(\Omega_{a}^{2} \partial_{s}^{2}+B^{2}\right) U_{a}=\lambda U_{a}^{3} .
$$

We formally expand the solution and frequency:

$$
\begin{aligned}
& U_{a}=a U_{1}+a^{3} U_{3}+\cdots . \\
& \Omega_{a}=\Omega+a^{2} \Omega_{2}+\cdots .
\end{aligned}
$$

Substitution of (1.32) into (1.31) and assembling terms according to their order in $a$, one gets a hierarchy of equations beginning with

$$
\begin{aligned}
& \mathcal{O}\left(a^{1}\right):\left(\Omega^{2} \partial_{s}^{2}+B^{2}\right) U_{1}=0 \\
& \mathcal{O}\left(a^{3}\right):\left(\Omega^{2} \partial_{s}^{2}+B^{2}\right) U_{3}=\lambda U_{1}^{3}-2 \Omega \Omega_{2} \partial_{s}^{2} U_{1}
\end{aligned}
$$

\footnotetext{
${ }^{2}$ In [40], breather type solutions have been constructed for the discrete sine-Gordon equation, where $\partial_{x}^{2}$, is replaced by its discretization on a sufficiently coarse lattice. Also, a generalization of the notion of breather has been considered in the geometric context of wave maps [55].
} 
Equation (1.33) has a solution

$$
U_{1}(x, s)=\cos s \varphi(x) .
$$

Substitution into (1.34) gives the following explicit equation for $U_{3}$ :

$$
\left(\Omega^{2} \partial_{s}^{2}+B^{2}\right) U_{3}=\left(\frac{3 \lambda}{4} \varphi^{3}+2 \Omega \Omega_{2} \varphi\right) \cos s+\frac{\lambda}{4} \varphi^{3} \cos 3 s
$$

We now express $U_{3}$ in the form $U_{3}=U_{3}^{(1)}+U_{3}^{(2)}$, where

$$
\begin{aligned}
& \left(\Omega^{2} \partial_{s}^{2}+B^{2}\right) U_{3}^{(1)}=\left(\frac{3 \lambda}{4} \varphi^{3}+2 \Omega \Omega_{2} \varphi\right) \cos s \\
& \left(\Omega^{2} \partial_{s}^{2}+B^{2}\right) U_{3}^{(2)}=\frac{\lambda}{4} \varphi^{3} \cos 3 s .
\end{aligned}
$$

Since the inhomogeneous term in (1.37) is nonresonant with the continuous spectrum of $B^{2}$, one can find a $2 \pi$-periodic solution $U_{3}^{(1)}$ provided the right hand side is $L^{2}\left(S_{2 \pi}^{1} \times \mathbb{R}^{3}\right)$ - orthogonal to the adjoint zero mode: $\cos s \varphi(x)$. This latter condition uniquely determines $\Omega_{2}$. Note however that the right hand side of (1.38) is resonant with continuous spectrum of $B^{2}$ if $3 \Omega>m$. To compute the obstruction to solvability, seek a solution of (1.38) of the form $U_{3}^{(2)}=\cos 3 s F$. Then,

$$
\left(B^{2}-9 \Omega^{2}\right) F=\frac{\lambda}{4} \varphi^{3}
$$

and thus we expect to find $F \in L^{2}$ if and only if the component of $\varphi^{3}$ in the direction of the generalized eigenfunction at frequency $3 \Omega$ of the operator $B$ vanishes. Therefore, if the nonlinear Fermi golden rule (1.8) holds, our formal expansion in $L^{2}\left(S_{2 \pi}^{1} \times \mathbb{R}^{3}\right)$ breaks down. The relation with our work is that we prove that this obstruction to solvability, in fact, implies the radiative behavior of solutions described in our main theorem.

\section{Linear analysis}

In this section we summarize the tools of linear analysis employed in this paper. 


\subsection{Estimates for the (free) Klein Gordon equation}

We begin by considering the Cauchy problem for the linear KleinGordon equation for a function $u(x, t), x \in \mathbb{R}^{n}, t \neq 0$.

$$
\begin{aligned}
\partial_{t}^{2} u & =\left(\Delta-m^{2}\right) u=-B_{0}^{2} u \\
u(x, 0) & =u_{0}(x), \quad \partial_{t} u(x, 0)=u_{1}(x) .
\end{aligned}
$$

There exist operators $E_{0}^{(0)}(t)$ and $E_{1}^{(0)}(t)$ such that

$$
u(x, t)=E_{0}^{(0)}(t) u_{0}+E_{1}^{(0)}(t) u_{1}
$$

We write

$$
\begin{aligned}
& E_{0}^{(0)}(t) f=\cos B_{0} t f, \text { and } \\
& E_{1}^{(0)}(t) g=\frac{\sin B_{0} t}{B_{0}} g
\end{aligned}
$$

where $\left(\omega(k)=\sqrt{m^{2}+k^{2}}\right)$ :

$$
\begin{aligned}
& E_{0}^{(0)}(t) f=\int \cos \omega(k) t \hat{f}(k) \mathrm{e}^{\mathrm{i} k \cdot x} d x \\
& E_{1}^{(0)}(t) g=\int \frac{\sin \omega(k) t}{\omega(k)} \hat{g}(k) \mathrm{e}^{\mathrm{i} k \cdot x} d x .
\end{aligned}
$$

The first result we cite is proved using stationary phase methods; see [5]. See [36] for another approach to decay estimates for (2.1). Let $W^{s, p}\left(\mathbb{R}^{n}\right)$ denote the Sobolev space of functions with derivatives of order $\leq s$ in $L^{p}$.

Theorem 2.1. Let $1<p \leq 2, \frac{1}{p}+\frac{1}{p^{\prime}}=1, \delta \equiv \frac{1}{2}-\frac{1}{p^{\prime}}$ and $0 \leq \theta \leq 1$. Then,

(a) if $\delta(n+1+\theta) \leq v+s$, we have for $v=0,1$ :

$$
\begin{aligned}
\left\|E_{v}^{0}(t) g\right\|_{p^{\prime}} & \leq K(t)\|g\|_{s, p}, \quad t \geq 0, \text { where } \\
K(t) & =C t^{-(n-1-\theta) \delta}, \quad 0<t \leq 1, \\
& =C t^{-(n-1+\theta) \delta}, \quad t \geq 1 .
\end{aligned}
$$

(b) If $s \geq\left(1 / 2-1 / p^{\prime}\right)(n+2)-1$, then for $t \geq 1$ the Schrödingerlike $L^{p^{\prime}}$ decay rate, $t^{-n\left(\frac{1}{2}-\frac{1}{p^{\prime}}\right)}$, holds in (2.7). 
In subsequent sections, we shall use some specific consequences of this result.

Corollary 2.1. Consider the linear Klein Gordon equation (2.1) in dimension $n=3$. Then,

$$
\begin{aligned}
& \left\|E_{1}^{(0)}(t) g\right\|_{8} \leq C t^{-\frac{9}{8}}\|g\|_{1, \frac{8}{7}} \quad t \geq 1 \\
& \left\|E_{1}^{(0)}(t) g\right\|_{8} \leq C t^{-\frac{3}{8}}\|g\|_{1, \frac{8}{7}} \quad 0<t \leq 1 \\
& \left\|E_{v}^{(0)}(t) g\right\|_{4} \leq C t^{-\frac{1}{2}}\|g\|_{1, \frac{4}{3}}, \quad t>0, \quad v=0,1 .
\end{aligned}
$$

Proof. Estimates (2.8) and (2.9) follow from the theorem with the choice of parameters: $p^{\prime}=8, s=1, n=3$, and $\theta=1$. Estimate (2.10) follows from the theorem with the choice of parameters: $p^{\prime}=4, s=1, n=3$ and $\theta=0$.

\subsection{Estimates for the Klein Gordon equation with a potential}

We now consider the Cauchy problem for the linear Klein-Gordon equation with a potential

$$
\partial_{t}^{2} u=\left(\Delta-m^{2}-V(x)\right) u=-B^{2} u
$$

where $B^{2}$ is positive and self-adjoint.

We write the solution to the initial value problem for (2.11) with initial data (2.2) as:

$$
\begin{aligned}
u(x, t)= & E_{0}(t) u_{0}+E_{1}(t) u_{1}, \quad \text { where } \\
& E_{0}(t) f=\cos (B t) f, \text { and } \\
& E_{1}(t) g=\frac{\sin (B t)}{B} g .
\end{aligned}
$$

One expects that estimates of the type appearing in Theorem 2.1 and Corollary 2.1 will hold as well for $E_{0}(t)$ and $E_{1}(t)$ restricted to $\mathbf{P}_{c} L^{2}$, the continuous spectral part of $H=B^{2}$. One can relate functions of the operator $H$, on its continuous spectral part, to functions of $H_{0}=B_{0}^{2}$ using wave operators. Let

$$
W_{+}=\text {strong }-\lim _{t \rightarrow \infty} \mathrm{e}^{\mathrm{i} t H} \mathrm{e}^{-\mathrm{i} t H_{0}} .
$$

Wave operators relate $H_{0}$ to $H$ on $\mathbf{P}_{c} L^{2}$ via the intertwining property: 


$$
H=W_{+} H_{0} W_{+}^{*} \quad \text { on } \mathbf{P}_{\mathrm{c}} L^{2} .
$$

K. Yajima [72] has proved the $W^{k, p}\left(\mathbb{R}^{n}\right)$ boundedness of wave operators for the Schrödinger equation. A consequence of this work is the following result for spatial dimension $n=3$ :

Theorem 2.2. Let $H=-\Delta+V$, where $V(x)$ be real-valued function on $\mathbb{R}^{3}$ satisfying the following hypotheses, which are satisfied by smooth and sufficiently rapidly decaying potentials: For any $|\alpha| \leq \ell$ there is a constant $C_{\alpha}$ such that

$$
\left|\frac{\partial^{\alpha} V}{\partial x^{\alpha}}(x)\right| \leq C_{\alpha}\langle x\rangle^{-\delta}, \quad \delta>5
$$

Additionally, assume that 0 is neither an eigenvalue nor a resonance of $H$; see [72], [31]. If $0 \leq k, k^{\prime} \leq l$ and $1 \leq p, p^{\prime} \leq \infty$, then there exists a constant $C>0$ such that for any Borel function $f$ on $\mathbb{R}^{1}$ we have

$$
\begin{aligned}
C^{-1}\left\|f\left(H_{0}\right)\right\|_{B\left(W^{k, p}, W^{k^{\prime}, p^{\prime}}\right)} & \leq\left\|f(H) \mathbf{P}_{c}(H)\right\|_{B\left(W^{k, p}, W^{k^{\prime}, p^{\prime}}\right)} \\
& \leq C\left\|f\left(H_{0}\right)\right\|_{B\left(W^{k, p}, W^{k^{\prime}, p^{\prime}}\right)} .
\end{aligned}
$$

Here, $\mathbf{P}_{c}(H)$ denotes the projection onto the continuous spectral part of the operator $H$.

Remark. In our applications, we shall use Theorem 2.2 with $|l| \leq 2$.

Theorem 2.2 implies that the dispersive estimates for the free Klein Gordon group carry over to the operators $E_{0}(t)$ and $E_{1}(t)$ restricted to the continuous spectral part of $B$.

Theorem 2.3. If $g \in$ Range $\mathbf{P}_{c}(H)$, then each of the estimates of Theorem 2.1 and Corollary 2.1 hold with $E_{j}^{(0)}$ replaced by $E_{j}$.

\subsection{Singular resolvents and time decay}

As discussed in the introduction, the damping term in the effective nonlinear oscillator (1.26), is related to the evaluation of a singular limit of the resolvent as the eigenvalue parameter approaches the continuous spectrum. To ensure that the correction terms to the nonlinear oscillator (1.26) can be treated perturbatively, we require local decay estimates for the operator $\mathrm{e}^{\mathrm{i} B t}(B-\Lambda \pm \mathrm{i} 0)^{-1}$, where $\Lambda$ is a point in the interior of the continuous spectrum of $B(\Lambda>m)$. Such 
estimates are analogous to those used by the authors in recent work on a time dependent approach to the quantum resonance problem [62].

We begin with a proposition, which is essentially a restatement of Theorem 2.3 for $E_{j}(t)$, and is proved the same way.

Proposition 2.1. $\left(W^{s, p}\right.$ estimates for $\mathrm{e}^{\mathrm{i} B t}$ ) Assume that $V(x)$ satisfies the hypotheses of Theorem $2.2(n=3)$; see [72] for hypotheses for general space dimension, $n \geq 3$. Let $p$ and $p^{\prime}$ be as in Theorem 2.1 and let $l \geq s \geq\left(\frac{1}{2}-\frac{1}{p^{\prime}}\right)(n+2)$, where $l$ is as in Theorem 2.2. Then,

$$
\left\|\mathrm{e}^{\mathrm{i} B t} \mathbf{P}_{\mathrm{c}} \psi\right\|_{p^{\prime}} \leq C|t|^{-n\left(\frac{1}{2}-\frac{1}{p^{\prime}}\right)}\|\psi\|_{s, p}, \quad t \neq 0
$$

The next proposition is the "singular" resolvent estimate we shall require in Section 7. Let $\sigma_{*}(n)=\max \left\{\frac{n}{2}, 2+\frac{2 n}{n+2}\right\}$.

Proposition 2.2. Assume the hypotheses of Proposition 2.1. Additionally, assume that $V(x)$ satisfies hypothesis (V2) of Theorem 1.1. Also, let $\sigma>\sigma_{*}(n)$. Then, for any point $\Lambda(\Lambda>m)$ in the continuous spectrum of $B$ we have for $l=0,1,2$ :

$$
\begin{gathered}
\left\|\langle x\rangle^{-\sigma} \mathrm{e}^{\mathrm{i} B t}(B-\Lambda+\mathrm{i} 0)^{-l} \mathbf{P}_{\mathrm{c}}\langle x\rangle^{-\sigma} \psi\right\|_{2} \leq C\langle t\rangle^{-\frac{2 n}{n+2}}\|\psi\|_{1,2}, \quad t>0, \\
\left\|\langle x\rangle^{-\sigma} \mathrm{e}^{\mathrm{i} B t}(B-\Lambda-i 0)^{-l} \mathbf{P}_{\mathrm{c}}\langle x\rangle^{-\sigma} \psi\right\|_{2} \leq C\langle t\rangle^{-\frac{2 n}{n+2}}\|\psi\|_{1,2}, \quad t<0,
\end{gathered}
$$

Proof. We prove the estimate (2.17) for the case of the $t>0$. For the case, $t<0$, the same argument with simple modifications applies.

Let $g_{\Delta}=g_{\Delta}(B)$ denote a smooth characteristic function of an open interval, $\Delta$ which contains $\Lambda$ and is contained in the continuous spectrum of $B$. Also, let $\bar{g}_{\Delta} \equiv 1-g_{\Delta}$. We then write, for $l=1,2$ :

$$
\begin{aligned}
\mathrm{e}^{\mathrm{i} B t}(B-\Lambda+i \varepsilon)^{-l} \mathbf{P}_{\mathrm{c}}= & \mathrm{e}^{\mathrm{i} B t} g_{\Delta}(B)(B-\Lambda+\mathrm{i} \varepsilon)^{-l} \mathbf{P}_{\mathrm{c}} \\
& +\mathrm{e}^{\mathrm{i} B t} \bar{g}_{\Delta}(B)(B-\Lambda+\mathrm{i} \varepsilon)^{-l} \mathbf{P}_{\mathrm{c}} \equiv S_{1}^{\varepsilon}(t)+S_{2}^{\varepsilon}(t) .
\end{aligned}
$$

We now estimate the operators $S_{j} \equiv \lim _{\varepsilon \rightarrow 0} S_{j}^{\varepsilon}, j=1,2$ individually. Estimation of $S_{1}(t)$ :

Consider the case $l=2$; the case $l=1$ is simpler. First, we note that: 


$$
\begin{aligned}
& \langle x\rangle^{-\sigma} S_{1}^{\varepsilon}(t)\langle x\rangle^{-\sigma} \\
& =-\mathrm{e}^{\mathrm{i} t(\Lambda-i \varepsilon)} \int_{t}^{\infty} d s \int_{s}^{\infty} d \tau\langle x\rangle^{-\sigma} \mathrm{e}^{\mathrm{i} \tau(B-\Lambda+i \varepsilon)} g_{\Delta}(B) \mathbf{P}_{\mathrm{c}}\langle x\rangle^{-\sigma}
\end{aligned}
$$

Now we claim that the operator in the integrand satisfies the estimate:

$$
\left\|\langle x\rangle^{-\sigma} \mathrm{e}^{\mathrm{i} \tau(B-\Lambda+\mathrm{i} \varepsilon)} g_{\Delta}(B) \mathbf{P}_{\mathrm{c}}\langle x\rangle^{-\sigma}\right\|_{\mathscr{B}\left(L^{2}\right)} \leq C \frac{e^{-\varepsilon \tau}}{\langle\tau\rangle^{r}}
$$

for $r<\sigma$. The desired estimate on $S_{1}^{\varepsilon}$ then follows by use of (2.20) in (2.19) and that $\sigma>\sigma_{*}(n)$.

It is simple to see that (2.20) is expected. For, suppose that instead of $B \mathbf{P}_{c}$ we had $B_{0}$. Let $\omega(k)=\sqrt{m^{2}+k^{2}}$, the dispersion relation of $B_{0}$. Then, setting $L=\left(\mathrm{i} \partial_{k_{j}} \omega\right)^{-1} \partial_{k_{j}}$, and using that $\left|\nabla_{k} \omega\right| \neq 0$ on the support of $g_{\Delta}$, we get

$$
\begin{aligned}
\mathrm{e}^{\mathrm{i} B_{0} t} g_{\Delta}\left(B_{0}\right) f & =\int \mathrm{e}^{\mathrm{i} \omega(k) t} \mathrm{e}^{\mathrm{i} k \cdot x} g_{\Delta}(k) \hat{f}(k) d k \\
& =t^{-r} \int L^{r}\left(\mathrm{e}^{\mathrm{i} \omega(k) t}\right) \mathrm{e}^{\mathrm{i} k \cdot x} g_{\Delta}(k) \hat{f}(k) d k \\
& =t^{-r} \int \mathrm{e}^{\mathrm{i} \omega(k) t}\left(L^{\dagger}\right)^{r}\left(\mathrm{e}^{\mathrm{i} k \cdot x} g_{\Delta}(k) \hat{f}(k)\right) d k .
\end{aligned}
$$

Estimation of $\langle x\rangle^{-\sigma} \mathrm{e}^{\mathrm{i} B_{0} t} g_{\Delta}\left(B_{0}\right) f$ in $L^{2}$ by Fourier transform methods, using that $|\nabla \omega|$ is bounded away from zero on the support of $g_{\Delta}$, we have that if $\sigma>\max \left\{\frac{n}{2}, 2\right\}$ and $r<\sigma$, then

$$
\left\|\langle x\rangle^{-\sigma} \mathrm{e}^{\mathrm{i} B_{0} t} g_{\Delta}\left(B_{0}\right) f\right\|_{2} \leq C\langle t\rangle^{-r}\left\|\langle x\rangle^{\sigma} f\right\|_{2} .
$$

Use of this estimate, in the above expression for $S_{1}^{\varepsilon}$ (with $B$ replaced by $B_{0}$ ) gives, for $l=0,1,2$ :

$$
\left\|\langle x\rangle^{-\sigma} \mathrm{e}^{\mathrm{i} B_{0} t}\left(B_{0}-\Lambda+\mathrm{i} 0\right)^{-l}\langle x\rangle^{-\sigma}\right\|_{\mathscr{B}\left(L^{2}\right)} \leq C\langle t\rangle^{-r+l} .
$$

Two approaches to proving an estimate of the type (2.22) and (2.23) for $\mathrm{e}^{\mathrm{i} B t} g_{\Delta}(B) \mathbf{P}_{\mathrm{c}}$ are as follows:

(a) One can "map" the estimate for $B_{0}$ to that for $B \mathbf{P}_{\mathrm{c}}$ using the wave operator $W_{+}$. In this approach we would need to derive estimates for $W_{+} g_{\Delta}\left(B_{0}\right)$ in weighted $L^{2}$ spaces, or alternatively

(b) One can use the approach based on the "Mourre estimate", developed in quantum scattering theory. This approach is more in the 
spirit of energy estimates for partial differential equations and does not require the use of wave operators.

Here, we shall follow the latter approach. Time decay estimates like (2.22) are a consequence of an approach to minimal velocity estimates of Sigal and Soffer [58], which were proved using the ideas of Mourre [43]; see also Perry, Sigal \& Simon [48]. For our application to the operator $\mathrm{e}^{-\mathrm{i} B t} g_{\Delta}(B)$, we shall refer to special cases of results stated in Skibsted [59] and Debiévre, Hislop \& Sigal [20]. (A0)

We first introduce some definitions and assumptions:.

(a) Let $N \geq 2$ and $g_{\Delta} \in C_{0}^{\infty}$, be a smooth characteristic function which is equal to one on the open interval $\Delta$.

(b) Let $H$ and $A$ denote self adjoint operators on a Hilbert space $\mathscr{H}$. Assume $H$ is bounded below. Let $\mathscr{D}$ denote the domain of $A$ and $\mathscr{D}(H)$ the domain of $H$. Assume $\mathscr{D} \cap \mathscr{D}(H)$ is dense in $\mathscr{D}(H)$, and let $\langle A\rangle \equiv\left(I+|A|^{2}\right)^{\frac{1}{2}}$.

(A1) Let $\operatorname{ad}_{A}^{0}(H)=H$. For $1 \leq k \leq N_{*}$, define iteratively the commutator form

$$
\mathrm{i}^{k} \operatorname{ad}_{A}^{k}(H)=\mathrm{i}\left[\mathrm{i}^{k-1} \operatorname{ad}_{A}^{k-1}(H), A\right]
$$

on $\mathscr{D} \cap \mathscr{D}(H)$. Assume that for $1 \leq k \leq N_{*}, \mathrm{i}^{k} \operatorname{ad}_{A}^{k}(H)$ extends to a symmetric operator with domain $\mathscr{D}(H)$, where $N_{*}=\left[N+\frac{3}{2}\right]+1$.

(A2) For $|s|<1, \mathrm{e}^{\mathrm{i} A s}: \mathscr{D}(H) \mapsto \mathscr{D}(H)$ and $\sup _{|s|<1}\left\|H \mathrm{e}^{\mathrm{i} A s} \psi\right\|_{\mathscr{H}}$ $<\infty$, for any $\psi \in \mathscr{D}(H)$.

(A3) Mourre estimate:

$$
g_{\Delta}(H) \mathrm{i}[H, A] g_{\Delta}(H) \geq \theta g_{\Delta}(H)^{2}
$$

for some $\theta>0$.

Under the above assumptions, we have, via Theorem 2.4 of [59], the following:

Theorem 2.4. Assume conditions $(A 0)-(A 3)$. Then, for all $\varepsilon_{1}>0$ and $t>0$

$$
\left\|F\left(\frac{A}{t}<\theta\right) \mathrm{e}^{-\mathrm{i} H t} g_{\Delta}(H)\langle A\rangle^{-\frac{N}{2}} \psi\right\|_{\mathscr{H}} \leq C\langle t\rangle^{-\frac{N}{2}+\varepsilon_{1}}\|\psi\|_{\mathscr{H}},
$$

where $\theta$ is as in (2.25). 
To prove this theorem we set $A(\tau)=A-b \tau$, where $\tau \equiv t+1$. Then, for any $b<\theta$, we have by (2.25) that the Heisenberg derivative, $D A(\tau) \equiv \partial_{t} A(\tau)+\mathrm{i}[H, A(\tau)]$, satisfies

$$
g_{\Delta} D A(\tau) g_{\Delta}=g_{\Delta}(\mathrm{i}[H, A]-b) g_{\Delta} \geq(\theta-b) g_{\Delta}^{2} .
$$

The result now follows by an application of Theorem 2.4 of [59].

Remark. The hypotheses of Theorem 2.4 can be relaxed [29] to the following two conditions: (i) the operator $g_{\Delta}(H) \operatorname{ad}_{A}^{n}(H) g_{\Delta}(H)$ can be extended to a bounded operator on $\mathscr{H}$ for $n=0,1,2 \ldots,\left[\frac{N}{2}+1\right]+1$, and (ii) the Mourre estimate, (2.25).

We shall apply Theorem 2.4 for the cases $H=B=$ $\sqrt{-\Delta+m^{2}+V(x)}, A=(x \cdot p+p \cdot x) / 2, p=-\mathrm{i} \nabla_{x}$ and $\mathscr{H}=L^{2}$. Before verifying the hypotheses of Theorem 2.4, we show how this theorem is used to derive the desired estimates on $\mathrm{e}^{\mathrm{i} B t} g_{\Delta}(B)$. With the application of Theorem 2.4 in mind, we estimate the norm of $\langle x\rangle^{-\sigma} \mathrm{e}^{\mathrm{i} B t} g_{\Delta}(B)$ by decomposing it into the spectral sets on which $A / t$ is less than and greater than or equal to $\theta$. Let $\Delta_{1}$ denote an interval containing $\Delta$ and denote by $\mathscr{C}$ the operator that associates to a function $f$ its complex conjugate $\bar{f}$. Furthermore, note that $\mathrm{e}^{\mathrm{i} B t}=\mathscr{C} \mathrm{e}^{-\mathrm{i} B t} \mathscr{C}$ and $\mathscr{C} g(B)=g(B) \mathscr{C}$, if $g$ is real-valued on the spectrum of $B$. Then we find,

$$
\begin{aligned}
\left\|\langle x\rangle^{-\sigma} \mathrm{e}^{\mathrm{i} B t} g_{\Delta}(B) \psi\right\|_{2}= & \left\|\langle x\rangle^{-\sigma} g_{\Delta_{1}}(B) \mathrm{e}^{\mathrm{i} B t} g_{\Delta}(B) \psi\right\|_{2} \\
= & \left\|\langle x\rangle^{-\sigma} g_{\Delta_{1}}(B) \mathscr{C} \mathrm{e}^{-\mathrm{i} B t} \mathscr{C} g_{\Delta}(B) \psi\right\|_{2} \\
= & \left\|\langle x\rangle^{-\sigma} g_{\Delta_{1}}(B)\langle A\rangle^{\sigma} \cdot\langle A\rangle^{-\sigma} \mathrm{e}^{-\mathrm{i} B t} g_{\Delta}(B) \bar{\psi}\right\|_{2} \\
\leq & \left\|\langle x\rangle^{-\sigma} g_{\Delta_{1}}(B)\langle A\rangle^{\sigma}\right\|_{\mathscr{B}\left(L^{2}\right)} \cdot\left\|\langle A\rangle^{-\sigma} \mathrm{e}^{-\mathrm{i} B t} g_{\Delta}(B) \bar{\psi}\right\|_{2} \\
\leq & C\left\|\langle A\rangle^{-\sigma} \mathrm{e}^{-\mathrm{i} B t} g_{\Delta}(B) \bar{\psi}\right\|_{2} \\
\leq & C_{1}\left\|F\left(\frac{A}{t}<\theta\right)\langle A\rangle^{-\sigma} \mathrm{e}^{-\mathrm{i} B t} g_{\Delta}(B) \bar{\psi}\right\|_{2} \\
& +C_{2}\left\|F\left(\frac{A}{t} \geq \theta\right)\langle A\rangle^{-\sigma} \mathrm{e}^{-\mathrm{i} B t} g_{\Delta}(B) \bar{\psi}\right\|_{2}
\end{aligned}
$$

We have used here that, with $A$ defined as above, the $L^{2}$ operator norm of $\langle x\rangle^{-\sigma} g_{\Delta_{1}}(B)\langle A\rangle^{\sigma}$ is bounded; see [48]. By Theorem 2.4, the first term on the right hand side of (2.28) can be estimated from above by: 


$$
\begin{aligned}
& C_{1}\left\|F\left(\frac{A}{t}<\theta\right)\langle A\rangle^{-\sigma} \mathrm{e}^{-\mathrm{i} B t} g_{\Delta}(B) g_{\Delta_{1}}(B) \bar{\psi}\right\|_{2} \\
& \quad \leq C_{1} C\langle t\rangle^{-\frac{N}{2}+\varepsilon_{1}}\left\|\langle A\rangle^{\frac{N}{2}} g_{\Delta_{1}}(B)\langle x\rangle^{-\frac{N}{2}}\right\|_{\mathscr{B}\left(L^{2}\right)}\left\|\langle x\rangle^{\frac{N}{2}} \psi\right\|_{2} .
\end{aligned}
$$

Since $\theta t>0$, the second term is bounded as follows:

$$
C_{2}\left\|F\left(\frac{A}{t} \geq \theta\right)\langle A\rangle^{-\sigma} \mathrm{e}^{-\mathrm{i} B t} g_{\Delta}(B) \bar{\psi}\right\|_{2} \leq C\langle t\rangle^{-\sigma}\|\psi\|_{2} .
$$

From (2.28), (2.29) and (2.30), we have

$$
\left\|\langle x\rangle^{-\sigma} \mathrm{e}^{\mathrm{i} B t} g_{\Delta}(B) \psi\right\|_{2} \leq C\left(\langle t\rangle^{-\sigma}+\langle t\rangle^{-\frac{N}{2}+\varepsilon_{1}}\right)\left\|\langle x\rangle^{\frac{N}{2}} \psi\right\|_{2} .
$$

Use of (2.28) in a direct estimation of (2.19) gives:

$$
\left\|\langle x\rangle^{-\sigma} S_{1}^{\varepsilon}(t)\langle x\rangle^{-\sigma}\right\|_{\mathscr{B}\left(L^{2}\right)} \leq C\left(\langle t\rangle^{-\frac{N}{2}+2+\varepsilon_{1}}+\langle t\rangle^{-\sigma+2}\right) .
$$

We make the final choice of $N$ and $\sigma$ after estimation of $S_{2}^{\varepsilon}(t)$.

To complete the estimation of $S_{1}^{\varepsilon}(t)$, it remains to verify hypotheses (A0)-(A3). These hypotheses are known to hold for the operator $H=B^{2}=-\Delta+V+m^{2}$; see [18]. Hypothesis (A0) holds $H=B$ with domain $W^{1,2}\left(\mathbb{R}^{n}\right)$. Clearly hypothesis (A2) holds for $H=B$ since it holds for $H=B^{2}$. Hypothesis (A1) can be reduced to its verification for $B^{2}$ using the Kato square root formula [49]: for any $\psi \in \mathscr{D}\left(B^{2}\right)$ :

$$
B \psi=\pi^{-1} \int_{0}^{\infty} w^{-1 / 2} B^{2}\left(B^{2}+w\right)^{-1} \psi d w .
$$

To verify (A1) for the case $k=1$ we must show that $[A, B] B^{-1}$ is a bounded operator on $L^{2}$. Using (2.33) we have:

$$
[A, B] B^{-1}=\pi^{-1} \int_{0}^{\infty} w^{1 / 2}\left(B^{2}+w\right)^{-1}\left[A, B^{2}\right] B^{-1}\left(B^{2}+w\right)^{-1} d w
$$

Since

$$
\left[B^{2}, \mathrm{i} A\right]=2 B^{2}-x \cdot \nabla V-2\left(V+m^{2}\right),
$$

we have that 


$$
\left[B^{2}, \mathrm{i} A\right] B^{-1}=2 B-(x \cdot \nabla V+2 V) B^{-1}-2 m^{2} B^{-1}
$$

Substitution into (2.34) we get:

$$
\begin{aligned}
{[A, B] B^{-1}=} & \frac{2}{\pi} \int_{0}^{\infty} w^{\frac{1}{2}} B\left(B^{2}+w\right)^{-2} d w \\
& -\frac{1}{\pi} \int_{0}^{\infty}\left(B^{2}+w\right)^{-1}\left(x \cdot \nabla V+2 V-2 m^{2}\right) B^{-1}\left(B^{2}+w\right)^{-1} d w \\
\equiv & J_{1}+J_{2}
\end{aligned}
$$

The term $J_{1}$ can be rewritten as

$$
J_{1}=-\frac{2}{\pi} \int_{0}^{\infty} w^{\frac{1}{2}} \frac{d}{d w}\left(B^{2}+w\right)^{-1} d w B=I
$$

which follows from integration by parts and the formula $(2.33)$ with $\psi$ replaced by $B^{-1} \psi$. Using that $\left\|\left(B^{2}+w\right)^{-1}\right\| \leq\left(\Omega^{2}+w\right)^{-1}$,

$$
\left\|\left(x \cdot \nabla V+2 V-2 m^{2}\right) B^{-1}\right\| \leq\left\|x \cdot \nabla V+2 V-2 m^{2}\right\|_{\infty}\left\|B^{-1}\right\|_{\mathscr{B}\left(L^{2}\right)},
$$

and the hypotheses on $V$ we have that for some constant $C$ dependent on $V$,

$$
\left|J_{2}\right| \leq C \int_{0}^{\infty} w^{\frac{1}{2}}\left(\Omega^{2}+w\right)^{-2} d w<\infty
$$

The higher order commutators are handled in a similar manner; they are even simpler because each successive commutator results in an extra factor of $\left(B^{2}+w\right)^{-1}$.

This leaves us with verification of the Mourre estimate (A3) for $H=B$. Proposition 2.2 of [20] says that under hypotheses on $B^{2}$. verified in [18], that (A3) holds for $B$ as well.

\section{Estimation of $\mathrm{S}_{2}$ :}

In $S_{2}(t)$, the energy is localized away from $\Lambda$ so we seek to use the $W^{k, s}$ estimates of Proposition 2.1. Note that of the cases $l=1$ and 
$l=2$, the case $l=1$ is "worse" because $\bar{g}_{\Delta}$ localizes the energy away from the singularity at $\Lambda$, and the $l=1$ term has slower decay for large energy. We therefore carry out the estimation for $l=1$.

Let $q^{-1}+p^{-1}=1 / 2$ and $p^{-1}+p^{-1}=1$. Since $\sigma>\frac{n}{2},\langle x\rangle^{-\sigma} \in L^{q}$ and therefore:

$$
\begin{aligned}
& \left\|\langle x\rangle^{-\sigma} S_{2}^{\varepsilon}(t)\langle x\rangle^{-\sigma} f\right\|_{2} \\
& \quad \leq C\left\|\langle x\rangle^{-\sigma}\right\|_{\mathscr{B}\left(L^{p^{\prime}}, L^{2}\right)}\left\|\mathrm{e}^{\mathrm{i} B_{0} t}\left(B_{0}-\Lambda+\mathrm{i} 0\right)^{-1} \bar{g}_{\Delta}\left(B_{0}\right) W_{+}^{*}\langle x\rangle^{-\sigma} f\right\|_{p^{\prime}} \\
& \quad \leq C\left\|\mathrm{e}^{\mathrm{i} B_{0} t} B_{0}^{-1}\right\|_{\mathscr{B}\left(W^{1, p}, L^{p^{\prime}}\right)}\left\|\bar{g}_{\Delta}\left(B_{0}\right)\left(B_{0}-\Lambda+\mathrm{i} 0\right)^{-1} B_{0} W_{+}^{*}\langle x\rangle^{-\sigma} f\right\|_{1, p} \\
& \quad \leq C\left\|\mathrm{e}^{\mathrm{i} B_{0} t} B_{0}^{-1}\right\|_{\mathscr{B}\left(W^{1, p,}, L^{\prime}\right)}\left\|\bar{g}_{\Delta}\left(B_{0}\right)\left(B_{0}-\Lambda+\mathrm{i} 0\right)^{-1} B_{0} \cdot B_{0} W_{+}^{*}\langle x\rangle^{-\sigma} f\right\|_{p} \\
& \quad \leq C\left\|\mathrm{e}^{\mathrm{i} B_{0} t} B_{0}^{-1}\right\|_{\mathscr{B}\left(W^{1, p}, L^{\prime}\right)}\left\|\bar{g}_{\Delta}\left(B_{0}\right)\left(B_{0}-\Lambda+\mathrm{i} 0\right)^{-1} B_{0}\right\|_{B\left(L^{p}\right)}\left\|B_{0} W_{+}^{*}\langle x\rangle^{-\sigma} f\right\|_{p} .
\end{aligned}
$$

The three factors in (2.41) are estimated as follows:

(i) By part (a) of Theorem 2.1,

$$
\left\|\mathrm{e}^{\mathrm{i} B_{0} t} B_{0}^{-1}\right\|_{\mathscr{B}\left(W^{1, p}, L^{p^{\prime}}\right)} \leq C|t|^{-\frac{2 n}{n+2}}
$$

where $p=2-8 /(n+6)$ and $p^{\prime}=2(n+2) /(n-2)$.

(ii) $\left\|\bar{g}_{\Delta}\left(B_{0}\right)\left(B_{0}-\Lambda+\mathrm{i} 0\right)^{-1} B_{0}\right\|_{\mathscr{B}\left(L^{p}\right)}$ is bounded because $\bar{g}_{\Delta}(\mu)$ $(\mu-\Lambda+\mathrm{i} 0)^{-1} \mu$ is a multiplier on $L^{p}$ [64].

Using the boundedness of $W_{+}$on $W^{2, p}$ and that $\sigma>\max \left\{\frac{n}{2}, 2\right\}$, we have

$$
\begin{aligned}
\left\|B_{0} W_{+}^{*}\langle x\rangle^{-\sigma} f\right\|_{p} & \leq\left\|W_{+}^{*}\langle x\rangle^{-\sigma} f\right\|_{1, p} \\
& \leq\left\|W_{+}^{*}\right\|_{\mathscr{B}\left(W^{2, p}\right)}\left\|\langle x\rangle^{-\sigma} f\right\|_{1, p} \\
& \leq C\|f\|_{1,2} .
\end{aligned}
$$

Therefore,

$$
\left\|\langle x\rangle^{-\sigma} S_{2}^{\varepsilon}\langle x\rangle^{-\sigma} \psi\right\|_{2} \leq C\langle t\rangle^{-\frac{2 n}{n+2}}\|\psi\|_{1,2}
$$

We now combine the estimates of $S_{j}^{\varepsilon}(t), j=1,2$ to complete the proof. Combining (2.32) and (2.43) and taking $\varepsilon \downarrow 0$ yields

$$
\begin{aligned}
& \left\|\langle x\rangle^{-\sigma} \mathrm{e}^{\mathrm{i} B t}(B-\Lambda+\mathrm{i} 0)^{-1} \mathbf{P}_{c}\langle x\rangle^{-\sigma} \psi\right\|_{2} \\
& \quad \leq C\left(\langle t\rangle^{-\frac{N}{2}+2+\varepsilon_{1}}+\langle t\rangle^{-\sigma+2}+\langle t\rangle^{-\frac{2 n}{n+2}}\right)\|\psi\|_{1,2} .
\end{aligned}
$$


The estimates (2.17) now follows by taking $N$ and $\sigma$ such that

$$
\begin{aligned}
\sigma & \geq 2+\frac{2 n}{n+2} \\
\sigma & >\max \left\{\frac{n}{2}, 2\right\} \\
\frac{N}{2} & \geq 2+\frac{2 n}{n+2}+\varepsilon_{1} .
\end{aligned}
$$

The constraints on $\sigma$ are implied by the hypothesis $\sigma>\sigma_{*}(n)$. The remaining constraint holds if $N>8$. Since we have applied Theorem 2.4 in our estimation of $S_{1}^{\varepsilon}$, we need that (A1) hold with $N_{*}=\left[N+\frac{3}{2}\right]+1 \geq 10$.

This completes the proof of Proposition 2.2.

\section{Existence theory}

In this section we outline an existence theory for (1.1) with initial conditions $u(x, 0)=u_{0} \in W^{2,2}\left(\mathbb{R}^{3}\right)$ and $\partial_{t} u(x, 0)=u_{1} \in W^{1,2}\left(\mathbb{R}^{3}\right)$. We first reformulate the initial value problem and then introduce the hypotheses on the operator $H$. Regarding (1.1) as a perturbation of a linear constant coefficient equation, we first write the initial value problem as:

$$
\begin{aligned}
\partial_{t}^{2} u+B_{0}^{2} u & =-V u+\lambda f(u) . \\
u(x, 0) & =u_{0}(x) \\
\partial_{t} u(x, 0) & =u_{1}(x)
\end{aligned}
$$

Let

$$
\begin{aligned}
\mathbf{u} & =\left(\begin{array}{l}
u \\
v
\end{array}\right), \quad U(t)=\left(\begin{array}{cc}
\cos \left(B_{0} t\right) & \sin \left(B_{0} t\right) / B_{0} \\
-B_{0} \sin \left(B_{0} t\right) & \cos \left(B_{0} t\right)
\end{array}\right), \\
\mathbf{u}_{0} & =\left(\begin{array}{l}
u_{0} \\
u_{1}
\end{array}\right), \quad \mathbf{F}(\mathbf{u})=\left(\begin{array}{c}
0 \\
-V u+\lambda f(u)
\end{array}\right) .
\end{aligned}
$$

Then, the initial value problem can be reformulated as a system of first order equations:

$$
\partial_{t} \mathbf{u}=\left(\begin{array}{cc}
0 & 1 \\
-B_{0}^{2} & 0
\end{array}\right) \mathbf{u}+\mathbf{F}(\mathbf{u})
$$


We now follow the strategy of reformulating the problem of finding a solution of the initial value problem as the problem of finding a fixed point $\mathbf{u}$ of an appropriate mapping. In particular, we seek $\mathbf{u}$ in the space

$$
\mathbf{X}_{0}=W^{2,2}\left(\mathbb{R}^{3}\right) \times W^{1,2}\left(\mathbb{R}^{3}\right)
$$

satisfying

$$
\mathscr{A} \mathbf{u}=\mathbf{u}
$$

where

$$
\mathscr{A} \mathbf{u}(t) \equiv U(t) \mathbf{u}_{0}+\int_{0}^{t} U(t-s) F(\mathbf{u}) d s .
$$

For a discussion of the existence and low energy scattering for the case $V \equiv 0$, see [23], [65], [36] and references cited therein.

Hypotheses on $H=-\Delta+V$

(H1) $V \in W^{1, \infty}$

(H2) $H=B^{2}$ is a positive and self-adjoint operator

(H3) The semi-infinite interval, $\left[\mathrm{m}^{2}, \infty\right)$, consists of absolutely continuous spectrum of $H$.

(H4) $H$ has exactly one (simple) eigenvalue $\Omega^{2}$ satisfying $0<\Omega^{2}$ $<m^{2}$, with corresponding eigenfunction $\varphi \in L^{2},\|\varphi\|_{2}=1$.

(N) $f(u)=u^{3}+f_{4}(u), f_{4}(u)=\mathcal{O}\left(u^{4}\right)$ and $f(u)$ is smooth in a neighborhood of $u=0$.

These hypotheses are by no means the least stringent, but are sufficient for the present purposes.

Theorem 3.1 (Local existence theory). Consider the Cauchy problem for the nonlinear Klein Gordon equation (1.1) with initial data, $\mathbf{u}_{0}$ of class $\mathbf{X}_{0}$.

(a) There exists strictly positive number, $T_{\max }$ and $T^{\max }$ (forward and backward maximal times of existence) which depend on the $\mathbf{X}_{0}$ norm of the initial data, such that the initial value problem has a unique solution of class $C^{0}\left(\left(-T^{\max },+T_{\max }\right) ; \mathbf{X}_{0}\right)$ in the sense of the integral equation (3.4).

(b) For $t \in\left(-T^{\max },+T_{\max }\right)$ conservation of energy holds: $\mathscr{E}\left[u(\cdot, t), \partial_{t} u(\cdot, t)\right]=\mathscr{E}\left[u_{0}, u_{1}\right]$, where $\mathscr{E}$ is defined in equation (1.4).

(c) Either $T_{\max }$ in finite or $T_{\max }$ is infinite. If $T_{\max }$ is finite, then

$$
\lim _{t \uparrow T_{\max }}\|\mathbf{u}(\cdot, t)\|_{\mathbf{x}_{\mathbf{0}}}=\infty .
$$


The analogous statement holds with $T_{\max }$ replaced by $T^{\max }$ and $\lim _{t \uparrow T_{\max }}$ replaced by $\lim _{t \downarrow-T^{\max }}$.

We now sketch a proof of Theorem 3.1. We restrict our attention to the case $t \geq 0$; the proof is identical for $t \leq 0$. Furthermore, we shall, for simplicity, consider the case of the cubic nonlinearity: $f(u)=u^{3}$. Using hypothesis (H1) on $V$, it is simple to show, using the estimates of Corollary 2.1 , that for $T>0$ sufficiently small and depending essentially on the $\mathbf{X}_{0}$ norm of $\mathbf{u}_{0}$, the mapping $A$ maps a closed ball in $C^{0}\left([0, T) ; \mathbf{X}_{0}\right)$ to itself, and is a strict contraction. This ensures the existence of a unique fixed point, which is a $C^{0}\left([0, T) ; \mathbf{X}_{0}\right)$ solution of the initial value problem (3.1) in the sense of the integral equation (3.4). That the $\mathbf{X}_{0}$ norm must blow up if $T_{\max }$ is finite is a standard continuation argument based on the fixed point proof of part (a).

In Section 7, we study the asymptotic behavior of solutions as $t \rightarrow \pm \infty$. A consequence of this analysis and the argument below is that if $\mathbf{u}_{0}$ satisfies the more stringent hypothesis that the norm $\left\|\mathbf{u}_{0}\right\|_{\mathbf{x}}$ is sufficiently small, where

$$
\mathbf{X} \equiv\left(W^{2,2} \cap W^{2,1}\right) \times\left(W^{1,2} \cap W^{1,1}\right),
$$

Then $T_{\max }=T^{\max }=\infty$ and the solution decays to zero as dilineated in the statement of Theorem 1.1.

We reason as follows. A unique solution, $\mathbf{u}(t)$, exists locally in time and is continuous in $t$ with values in $\mathbf{X}_{0}$. It follows that for $|t|<T$, the $W^{1,4}$ and $L^{8}$ norms of $u(\cdot, t)$ are finite. Moreover, the solution satisfies energy conservation and therefore $\|u(t)\|_{W^{1,2}}+\|v(t)\|_{2}$ is bounded uniformly by a constant which is independent of $T$, and depends only on $\left\|u_{0}\right\|_{W^{1,2}}+\left\|v_{0}\right\|_{2}$. To ensure that the quantities estimated continue to be well defined and satisfy the 'a priori' estimates of Section 7 it suffices to show that $\|\mathbf{u}(t)\|_{\mathbf{x}_{0}}$ remains bounded as $t \uparrow T$. A direct and elementary estimation of the integral equation (3.4) yields the estimate:

$$
\begin{aligned}
\|\mathbf{u}(t)\|_{\mathbf{x}_{\mathbf{0}}} \leq & C\left(\left\|u_{0}\right\|_{W^{2,2}}+\left\|u_{1}\right\|_{W^{1,2}}\right) \\
& +C\left(\|V\|_{W^{1, \infty}}, \lambda\right) \int_{0}^{t}\left(\|u(s)\|_{W^{1,2}}+\|u(s)\|_{W^{1,2}}^{3}+\left\|u^{2}(s) \nabla u(s)\right\|_{2}\right) d s .
\end{aligned}
$$

The main tool used in obtaining (3.8) is the bound $\left\|E_{1}^{(0)}(t) \partial_{i}\right\|_{\mathscr{B}\left(L^{2}\right)}$ $\leq m^{-1}$. 
A simple consequence of the 'a priori' bound (7.52) and the decomposition of $u(t, \cdot)$ is that

$$
\|\nabla u(t)\|_{4}+\|u(t)\|_{8} \leq \tilde{C},|t|<T .
$$

The constant $\tilde{C}$, depends on the norm $\left\|\mathbf{u}_{0}\right\|_{\mathbf{x}}$. The first two terms in the integrand of (3.8) are uniformly bounded by observation of energy. Furthermore, this energy bound together with (3.9) implies a bound on the last term in the integrand of the estimate (3.8). It follows that $\|\mathbf{u}(t)\|_{\mathbf{x}_{0}}$ remains bounded as $t \uparrow T$. Therefore, given the estimates of Section 7 we have $T_{\max }=\infty$, and the decay of solutions.

A further consequence of the proof is:

Corollary 3.1. Under the hypotheses of Theorem 1.1, the solution of the initial value problem exists globally in $W^{2,2}\left(\mathbb{R}^{3}\right)$ and satisfies the estimate:

$$
\|u(t)\|_{W^{2,2}} \leq C\langle t\rangle
$$

\section{Isolation of the key resonances and formulation as coupled finite and infinite dimensional dynamical system}

Using the notation (1.5), the initial value problem for (1.1) can be rewritten as

$$
\begin{aligned}
& \partial_{t}^{2} u+B^{2} u=\lambda f(u), f(u)=u^{3} \\
& u(x, 0)=u_{0}(x), \partial_{t} u(x, 0)=u_{1}(x) .
\end{aligned}
$$

For small amplitude solutions, it is natural to decompose the solution as follows:

$$
\begin{aligned}
u(x, t) & =a(t) \varphi(x)+\eta(x, t), \\
(\eta(\cdot, t), \varphi) & =0 \quad \text { for all } \mathrm{t} .
\end{aligned}
$$

Substitution of (4.2) into (4.1) gives

$$
a^{\prime \prime} \varphi+\partial_{t}^{2} \eta+\Omega^{2} a \varphi+B^{2} \eta=\lambda f(a \varphi+\eta)
$$

We now implement (4.3). Taking the inner product of (4.4) with $\varphi$ gives

$$
a^{\prime \prime}+\Omega^{2} a=\lambda(\varphi, f(a \varphi+\eta))
$$


Let $\mathbf{P}_{\mathrm{c}}$ denote the projection onto the continuous spectral part of $B^{2}$, i.e.

$$
\mathbf{P}_{c} v \equiv v-(\varphi, v) \varphi .
$$

Then, since $\eta=\mathbf{P}_{c} \eta$, we have

$$
\partial_{t}^{2} \eta+B^{2} \eta=\lambda \mathbf{P}_{c} f(a \varphi+\eta)
$$

Equations (4.5)-(4.7) comprise a coupled dynamical system for the bound state and continuous spectral components (relative to $H=B^{2}$ ) of the solution $u$. The initial conditions for this system are given by (1.16).

Expansion of the cubic terms in (4.5-4.7) gives the system

$$
\begin{aligned}
a^{\prime \prime}+\Omega^{2} a & =\lambda\left[a^{3} \int \varphi^{4}+3 a^{2} \int \varphi^{3} \eta+3 a \int \varphi^{2} \eta^{2}+\int \varphi \eta^{3}\right] \\
\partial_{t}^{2} \eta+B^{2} \eta & =\lambda \mathbf{P}_{c}\left(a^{3} \varphi^{3}+3 a^{2} \varphi^{2} \eta+3 a \varphi \eta^{2}+\eta^{3}\right),
\end{aligned}
$$

with initial conditions

$$
\begin{gathered}
a(0)=\left(\varphi, u_{0}\right), \quad a^{\prime}(0)=\left(\varphi, u_{1}\right) \\
\eta(x, 0)=\mathbf{P}_{c} u_{0}, \quad \partial_{t} \eta(x, 0)=\mathbf{P}_{\mathbf{c}} u_{1} .
\end{gathered}
$$

We now locate the source of the key resonance. Equation (4.8) has a homogeneous solution which oscillates with frequencies $\pm \Omega$. Thus, to leading order, $\eta$ solves a driven wave equation containing the driving frequencies $\pm 3 \Omega$. If $9 \Omega^{2}>m^{2}$, then by $(H 3)$, we expect a resonant interaction with radiation modes of energy $3 \Omega \in \sigma_{\text {cont }}(H)$.

This resonant part of $\eta$, has its dominant effect on the $a$-oscillator in the term of (4.8), which is linear is $\eta$. Our goal is to derive from (4.8-4.9) an equivalent dynamical system which is of the type described in the introduction, but which is corrected by terms which decay sufficiently rapidly with time and which can therefore be treated perturbatively.

We first write

$$
\eta=\eta_{1}+\eta_{2}+\eta_{3}
$$

where $\eta_{1}(t)$ satisfies the linear dynamics with the given initial data:

$$
\partial_{t}^{2} \eta_{1}+B^{2} \eta_{1}=0, \quad \eta_{1}(x, 0)=\mathbf{P}_{c} u_{0}, \partial_{t} \eta_{1}(x, 0)=\mathbf{P}_{c} u_{1},
$$

and $\eta_{2}(t)$ is the leading order response 


$$
\partial_{t}^{2} \eta_{2}+B^{2} \eta_{2}=\lambda a^{3} \mathbf{P}_{c} \varphi^{3} ; \eta_{2}(x, 0)=0, \partial_{t} \eta_{2}(x, 0)=0 .
$$

Expansion (4.8) can now be written in an expanded form.

$$
\begin{array}{r}
a^{\prime \prime}+\Omega a=\lambda\left[a^{3} \int \varphi^{4}+3 a^{2} \int \varphi^{3}\left(\eta_{1}+\eta_{2}+\eta_{3}\right)\right. \\
\left.+3 a \int \varphi^{2} \eta^{2}+\int \varphi \eta^{3}\right] \equiv F(a, \eta)
\end{array}
$$

We expect the function $a(t)$ to consist of "fast oscillations", coming from the natural frequency $\Omega$ and its nonlinearly generated harmonics, and slow variations due to its small amplitude. We next extract from $a(t)$ the dominant "fast" oscillations of frequency $\Omega$ :

$$
a(t)=A \mathrm{e}^{\mathrm{i} \Omega t}+\bar{A} \mathrm{e}^{-\mathrm{i} \Omega t} .
$$

We then substitute (4.15) into (4.14) and impose the constraint:

$$
A^{\prime} \mathrm{e}^{\mathrm{i} \Omega t}+\vec{A}^{\prime} \mathrm{e}^{-\mathrm{i} \Omega t}=0 .
$$

Equation (4.14) then is reduced to the first order equation:

$$
A^{\prime}=(2 i \Omega)^{-1} \mathrm{e}^{-\mathrm{i} \Omega t} F(a, \eta),
$$

where $F(a, \eta)=F(A, \bar{A}, \eta, t)$. From (4.14) we have that $F(a, \eta)$ is the sum of the following terms:

$$
\begin{aligned}
F_{1}(a) & =\lambda a^{3} \int \varphi^{4} \\
F_{2}\left(a, \eta_{j}\right) & =3 \lambda a^{2} \int \varphi^{3} \eta_{j}, \quad j=1,2,3 \\
F_{3}(a, \eta) & =3 \lambda a \int \varphi^{2} \eta^{2} \\
F_{4}(\eta) & =\lambda \int \varphi \eta^{3} .
\end{aligned}
$$

The remainder of this section is primarily devoted to an (involved) expansion of $F_{2}\left(a, \eta_{2}\right)$. The terms of this expansion are of several types: (a) the resonant damping term, (b) terms of the same order whose net effect is a nonlinear phase correction and (c) higher order terms which are to be treated perturbatively in the asymptotic analysis at $t \rightarrow \pm \infty$. 


\section{Computation of $F_{2}\left(a, \eta_{2}\right)$}

We first break $\eta_{2}$ into a part containing the key resonance $\eta_{2}^{r}$ and nonresonant part $\eta_{2}^{n r}$.

\section{Proposition 4.1.}

$$
\eta_{2}=\eta_{2}^{r}+\eta_{2}^{n r}
$$

where

$$
\eta_{2}^{r}=\frac{\lambda}{2 i B} \mathrm{e}^{\mathrm{i} B t} \int_{0}^{t} \mathrm{e}^{-\mathrm{i} s(B-3 \Omega)} A^{3}(s) d s \mathbf{P}_{\mathrm{c}} \varphi^{3}
$$

and

$$
\begin{aligned}
\eta_{2}^{n r}= & \frac{\lambda}{2 i B} \mathrm{e}^{\mathrm{i} B t}\left[3 \int_{0}^{t} \mathrm{e}^{-\mathrm{i} s(B-\Omega)} A^{2}(s) \bar{A}(s) d s\right. \\
& +3 \int_{0}^{t} \mathrm{e}^{-\mathrm{i} s(B+\Omega)} \vec{A}^{2}(s) A(s) d s \\
& \left.+\int_{0}^{t} \mathrm{e}^{-\mathrm{i} s(B+3 \Omega)} \vec{A}^{3}(s) d s\right] \mathbf{P}_{\mathrm{c}} \varphi^{3} \\
& -\frac{\lambda}{2 i B} \mathrm{e}^{-\mathrm{i} B t}\left[\int_{0}^{t} \mathrm{e}^{\mathrm{i} s(B+3 \Omega)} A^{3}(s) d s\right. \\
& +3 \int_{0}^{t} \mathrm{e}^{\mathrm{i} s(B+\Omega)} A^{2}(s) \bar{A}(s) d s+3 \int_{0}^{t} \mathrm{e}^{\mathrm{i} s(B-\Omega)} \vec{A}^{2}(s) A(s) d s \\
& \left.+\int_{0}^{t} \mathrm{e}^{\mathrm{i} s(B-3 \Omega)} \bar{A}^{3}(s) d s\right] \mathbf{P}_{\mathrm{c}} \varphi^{3} \\
\equiv & \sum_{j=1} \eta_{2 j}^{n r}{ }^{3}
\end{aligned}
$$

The superscripts $r$ and $n r$ denote respectively a resonant contribution and nonresonant contribution. 
Proof. The solution of (4.13) can be expressed, using the variation of constants formula, as:

$$
\eta_{2}=\lambda \int_{0}^{t} \frac{\sin B(t-s)}{B} a^{3}(s) d s \mathbf{P}_{\mathrm{c}} \varphi^{3},
$$

Substitution of (4.15) for $a(s)$ and using the expansion of $\sin (B(t-s))$ in terms of the operators $\exp ( \pm \mathrm{i} B(t-s))$ leads to an expression for $\eta_{2}$ which is a sum of eight terms. The term $\eta_{2}^{r}$, as defined above, is anticipated to be the most important. The other seven terms are lumped together in $\eta_{2}^{n r}$.

We now focus on $\eta_{2}^{r}$. In order to study $\eta_{2}^{r}$ near the resonant point $3 \Omega$ in the continuous spectrum of $B$, we first introduce a regularization of $\eta_{2}^{r}$. For $\varepsilon>0$, let

$$
\eta_{2 \varepsilon}^{r} \equiv \frac{\lambda}{2 \mathrm{i} B} \mathrm{e}^{\mathrm{i} B t} \int_{0}^{t} \mathrm{e}^{-\mathrm{i} s(B-3 \Omega+i \varepsilon)} A^{3}(s) d s \mathbf{P}_{\mathrm{c}} \varphi^{3}
$$

Note that $\eta_{2}^{r}=\lim _{\varepsilon \rightarrow 0} \eta_{2 \varepsilon}^{r}$. The following result, proved using integration by parts, isolates the key (local in $t$ ) resonant term.

Proposition 4.2. For $\varepsilon \geq 0$,

$$
\begin{aligned}
\eta_{2 \varepsilon}^{r}= & \frac{\lambda}{2}[B(B-3 \Omega+\mathrm{i} \varepsilon)]^{-1} \mathrm{e}^{3 \mathrm{i} \Omega t} A^{3}(t) \mathrm{e}^{\varepsilon t} \mathbf{P}_{c} \varphi^{3} \\
& -\frac{\lambda}{2} A_{0}^{3}[B(B-3 \Omega+\mathrm{i} \varepsilon)]^{-1} \mathrm{e}^{\mathrm{i} B t} \mathbf{P} \varphi^{3} \\
& -\frac{3}{2} \lambda[B(B-3 \Omega+\mathrm{i} \varepsilon)]^{-1} \mathrm{e}^{\mathrm{i} B t} \int_{0}^{t} \mathrm{e}^{-\mathrm{i} s(B-3 \Omega+i \varepsilon)} A^{2} A^{\prime} d s \mathbf{P}_{\mathrm{c}} \varphi^{3} \\
= & \eta_{* \varepsilon}^{r}+\eta_{* \varepsilon}^{n r 1}+\eta_{* \varepsilon}^{n r 2}
\end{aligned}
$$

Remark: The choice $+\mathrm{i} \varepsilon$ in (4.22) is motivated by the fact that the operator

$$
(B-3 \Omega+\mathrm{i} 0)^{-1} \mathrm{e}^{\mathrm{i} B t}
$$


satisfies appropriate decay estimates $t \rightarrow \infty$; see Proposition 2.1. It follows that the limit as $\varepsilon \rightarrow 0^{+}$of the last two terms in (4.23) decay in time like $\langle t\rangle^{-1-\alpha}(\alpha>0)$; see Section 7.

We now use the above computation to obtain an expression for $F_{2}\left(a, \eta_{2}\right)$. First, from Proposition 4.1 we have

$$
\begin{aligned}
F_{2}\left(a, \eta_{2}\right) & =F_{2}\left(a, \eta_{2}^{r}\right)+F_{2}\left(a, \eta_{2}^{n r}\right) \\
& =\lim _{\varepsilon \rightarrow 0} F_{2}\left(a, \eta_{* \varepsilon}^{r}\right)+\lim _{\varepsilon \rightarrow 0} F_{2}\left(a, \eta_{* \varepsilon}^{n r 1}+\eta_{* \varepsilon}^{n r 2}\right)+F_{2}\left(a, \eta_{2}^{n r}\right), \\
& \equiv F_{2}\left(a, \eta_{*}^{r}\right)+F_{2}\left(a, \eta_{*}^{n r 1}+\eta_{*}^{n r 2}\right)+F_{2}\left(a, \eta_{2}^{n r}\right),
\end{aligned}
$$

where $F_{2}(a, \cdot)$ is defined in (4.18). We begin with the contribution to (4.17) coming from $F_{2}\left(a, \eta_{*}^{r}\right)$. What follows now is a detailed expansion of the term $F_{2}\left(a, \eta_{*}^{r}\right)$ and $F_{2}\left(a, \eta_{2}^{n r}\right)$. The terms $F_{2}\left(a, \eta_{*}^{n r 1}+\eta_{*}^{n r 2}\right)$ can be treated perturbatively by estimation of its magnitude; see Section 5 .

$$
\text { Computation of } F_{2}\left(a, \eta_{*}^{r}\right)
$$

Let

$$
\begin{aligned}
\Lambda & \equiv \lim _{\varepsilon \rightarrow 0}\left(\mathbf{P}_{\mathrm{c}} \varphi^{3}, \frac{1}{B} \frac{B-3 \Omega}{(B-3 \Omega)^{2}+\varepsilon^{2}} \mathbf{P}_{\mathrm{c}} \varphi^{3}\right) \\
& =\left(\mathbf{P}_{\mathrm{c}} \varphi^{3}, \frac{1}{B} P \cdot V \cdot \frac{1}{B-3 \Omega} \mathbf{P}_{\mathrm{c}} \varphi^{3}\right), \text { and } \\
\Gamma & \equiv \lim _{\varepsilon \rightarrow 0}\left(\mathbf{P}_{\mathrm{c}} \varphi^{3}, \frac{1}{B} \frac{\varepsilon}{(B-3 \Omega)^{2}+\varepsilon^{2}} \mathbf{P}_{\mathrm{c}} \varphi^{3}\right) \\
& =\frac{\pi}{3 \Omega}\left(\mathbf{P} \varphi^{3}, \delta(B-3 \Omega) \mathbf{P}_{\mathrm{c}} \varphi^{3}\right) \\
& =\frac{\pi}{3 \Omega}\left|\mathscr{F}_{c}\left[\varphi^{3}\right](3 \Omega)\right|^{2} .
\end{aligned}
$$

By hypothesis (1.8), $\Gamma>0$.

We now substitute the expression for $\eta_{* \varepsilon}^{r}$, given in (4.23) into the definition of $F_{2}\left(a, \eta_{* \varepsilon}^{r}\right)$ in (4.18). Passage to the limit, $\varepsilon \rightarrow 0$, and use of the distributional identity:

$$
(x \pm \mathrm{i} 0)^{-1} \equiv \lim _{\varepsilon \rightarrow 0}(x \pm \mathrm{i} \varepsilon)^{-1}=\mathrm{P} \cdot \mathrm{V} \cdot x^{-1} \mp \mathrm{i} \pi \delta(x)
$$

yields: 


\section{Proposition 4.3.}

$$
\begin{aligned}
F_{2}\left(a, \eta_{*}^{r}\right) & =\lim _{\varepsilon \rightarrow 0} F_{2}\left(a, \eta_{* \varepsilon}^{r}\right) \\
& =\frac{3}{2} \lambda^{2}(\Lambda-\mathrm{i} \Gamma)\left[|A|^{4} A \mathrm{e}^{\mathrm{i} \Omega t}+A^{5} \mathrm{e}^{5 \mathrm{i} \Omega t}+2|A|^{2} A^{3} \mathrm{e}^{3 \mathrm{i} \Omega t}\right] .
\end{aligned}
$$

We have completed the evaluation of the first term in (4.25). To calculate the contribution of (4.29) to the amplitude equation (4.17) we need only multiply (4.29) by $(2 \mathrm{i} \Omega)^{-1} \mathrm{e}^{-\mathrm{i} \Omega t}$. This gives

\section{Proposition 4.4.}

$$
(2 \mathrm{i} \Omega)^{-1} \mathrm{e}^{-\mathrm{i} \Omega t} F_{2}\left(a, \eta_{*}^{r}\right)=-\frac{3}{4} \frac{\lambda^{2}}{\Omega}(\mathrm{i} \Lambda+\Gamma)\left[|A|^{4} A+A^{5} \mathrm{e}^{4 \mathrm{i} \Omega t}+2|A|^{2} A^{3} \mathrm{e}^{2 \mathrm{i} \Omega t}\right] .
$$

The term $-\frac{3}{4} \frac{\lambda^{2}}{\Omega} \Gamma|A|^{4} A$ plays the role of a nonlinear damping; it drives the decay of $A$ and, in turn, that of $\eta$; see the discussion in the introduction.

$$
\text { Computation of } F_{2}\left(a, \eta_{2}^{n r}\right) \text { : }
$$

We now focus on $F_{2}\left(a, \eta_{2}^{n r}\right)$, the third term in (4.25). This requires a rather extensive, expansion of $\eta_{2}^{n r}=\sum_{j} \eta_{2 j}^{n r}$; see (4.20). From Proposition 4.3, we expect the dominant terms to be $\mathcal{O}\left(|A|^{5}\right)$. Our approach is now to make explicit all terms which are formally $\mathcal{O}\left(|A|^{5}\right)$ (anticipating that $A^{\prime}=\mathcal{O}\left(|A|^{3}\right), A^{\prime \prime}=\mathcal{O}\left(|A|^{5}\right)$ and $\left(|A|^{2}\right)^{\prime}=\mathcal{O}\left(|A|^{6}\right)$ ) and to treat the remainder as a perturbation which we shall later estimate to be of higher order. This expansion of $\eta_{2}^{n r}$ is presented in the following proposition which we prove using repeated integration by parts. As written, these expressions are formal. By the definition of $F_{2}\left(a, \eta_{2}^{n r}\right)$ we require that they hold when integrating against a rapidly decaying function, i.e. $\varphi^{3}$.

Proposition 4.5. The following expansions hold in $S^{\prime}$ :

$$
\begin{aligned}
\eta_{21}^{n r}= & \frac{\lambda}{2 B(B-\Omega)}|A|^{2} A \mathrm{e}^{\mathrm{i} t \Omega} \mathbf{P}_{\mathrm{c}} \varphi^{3}+\frac{3 \lambda}{2 \mathrm{i} B(B-\Omega)^{2}} \mathrm{e}^{\mathrm{i} t \Omega}\left(|A|^{2} A\right)^{\prime} \mathbf{P}_{\mathrm{c}} \varphi^{3} \\
& +\frac{3 \lambda \mathrm{e}^{\mathrm{i} B t}}{2 B(B-\Omega)}\left[\left|A_{0}\right|^{2} A_{0}+\left.\left(|A|^{2} A\right)^{\prime}\right|_{t=0}\right] \mathbf{P}_{\mathrm{c}} \varphi^{3} \\
& -\frac{3 \lambda}{2 \mathrm{i} B(B-\Omega)^{2}} \mathrm{e}^{\mathrm{i} B t} \int_{0}^{t} \mathrm{e}^{-\mathrm{i} s(B-\Omega)}\left(|A|^{2} A\right)^{\prime \prime} d s \mathbf{P}_{\mathrm{c}} \varphi^{3}
\end{aligned}
$$




$$
\begin{aligned}
\eta_{22}^{n r}= & \frac{3 \lambda}{2 B(B+\Omega)}|A|^{2} \bar{A} \mathrm{e}^{-\mathrm{i} t \Omega} \mathbf{P}_{\mathrm{c}} \varphi^{3} \\
& +\frac{3 \lambda}{2 \mathrm{i} B(B+\Omega)^{2}} \mathrm{e}^{-\mathrm{i} t \Omega}\left(\bar{A}^{2} A^{\prime}+2 \bar{A}^{\prime}|A|^{2}\right) \mathbf{P}_{\mathrm{c}} \varphi^{3} \\
& +\mathrm{e}^{\mathrm{i} B t}\left[-\frac{3}{2} \frac{\lambda}{2 B(B+\Omega)}\left|A_{0}\right|^{2} \bar{A}-\left.\frac{3 \lambda}{2 B(B+\Omega)}\left(|A|^{2} A\right)^{\prime}\right|_{t=0}\right] \mathbf{P}_{\mathrm{c}} \varphi^{3} \\
& -\frac{3 \lambda}{2 \mathrm{i} B(B+\Omega)^{2}} \mathrm{e}^{\mathrm{i} B t} \int_{0}^{t} \mathrm{e}^{-\mathrm{i} s(B+\Omega)}\left(|A|^{3} \bar{A}\right)^{\prime \prime}(s) d s \mathbf{P}_{\mathrm{c}} \varphi^{3} \\
\eta_{23}^{n r}= & \frac{\lambda \bar{A}(t)^{3}}{2 B(B+3 \Omega)} \mathrm{e}^{-3 \mathrm{i} t \Omega} \mathbf{P}_{\mathrm{c}} \varphi^{3}+\frac{3 \lambda}{2 \mathrm{i} B(B+3 \Omega)^{2}} \mathrm{e}^{-\mathrm{i} t 3 \Omega}\left(\bar{A}(t)^{3}\right)^{\prime} \mathbf{P}_{\mathrm{c}} \varphi^{3} \\
& -\frac{3}{2} \lambda \frac{\mathrm{e}^{\mathrm{i} B t}}{B(B+3 \Omega)}\left[\bar{A}_{0}^{3}+\left.\left(\bar{A}^{3}\right)^{\prime}\right|_{t=0}\right] \mathbf{P}_{\mathrm{c}} \varphi^{3} \\
& -\frac{3}{2} \frac{\lambda}{\mathrm{i} B(B+3 \Omega)^{2}} \mathrm{e}^{\mathrm{i} B t} \int_{0}^{t} \mathrm{e}^{-\mathrm{i} s(B+3 \Omega)}\left(\bar{A}(s)^{2}\right)^{\prime \prime} d s \mathbf{P}_{\mathrm{c}} \varphi^{3}
\end{aligned}
$$$$
\eta_{24}^{n r}=\frac{\lambda}{2 B(B+3 \Omega)} A^{3}(t) \mathrm{e}^{3 i i \Omega} \mathbf{P}_{\mathrm{c}} \varphi^{3}-\frac{3 \lambda}{2 \mathrm{i} B(B+3 \Omega)^{2}} \mathrm{e}^{3 \mathrm{i} t \Omega}\left(A^{3}\right)^{\prime} \mathbf{P}_{\mathrm{c}} \varphi^{3}
$$$$
-\frac{3}{2} \lambda \frac{\mathrm{e}^{-\mathrm{i} B t}}{B(B+3 \Omega)}\left[A_{0}^{3}+\left(A^{3}\right)_{t=0}^{\prime}\right] \mathbf{P}_{\mathrm{c}} \varphi^{3}
$$$$
+\frac{3 \lambda}{2 \mathrm{i} B(B+3 \Omega)^{2}} \mathrm{e}^{-\mathrm{i} B t} \int_{0}^{t} \mathrm{e}^{\mathrm{i} s(B+3 \Omega)}\left(A^{3}(s)\right)^{\prime \prime} d s \mathbf{P}_{\mathrm{c}} \varphi^{3}
$$

$$
\begin{aligned}
\eta_{25}^{n r}= & \frac{3 \lambda}{2 B(B+\Omega)}|A|^{2} A \mathrm{e}^{\mathrm{i} i \Omega} \mathbf{P}_{c} \varphi^{3}-\frac{3 \lambda}{2 \mathrm{i} B(B+\Omega)^{2}} \mathrm{e}^{\mathrm{i} t \Omega}\left(|A|^{2} A\right)^{\prime} \mathbf{P}_{\mathrm{c}} \varphi^{3} \\
& -\frac{3}{2} \lambda \frac{\mathrm{e}^{-\mathrm{i} B t}}{B(B+\Omega)}\left[\left|A_{0}\right|^{2} A_{0}+\left.\left(|A|^{2} A\right)^{\prime}\right|_{t=0}\right] \mathbf{P}_{\mathrm{c}} \varphi^{3} \\
& +\frac{3 \lambda}{2 \mathrm{i} B(B+\Omega)^{2}} \mathrm{e}^{-\mathrm{i} B t} \int_{0}^{t} \mathrm{e}^{\mathrm{i} s(B+\Omega)}\left(|A|^{2} A\right)^{\prime \prime} d s \mathbf{P}_{\mathrm{c}} \varphi^{3}
\end{aligned}
$$




$$
\begin{aligned}
\eta_{26}^{n r}= & \frac{3 \lambda}{2 B(B-\Omega)}|A|^{2} \bar{A} \mathrm{e}^{-\mathrm{i} t \Omega} \mathbf{P}_{\mathrm{c}} \varphi^{3}-\frac{3 \lambda}{2 \mathrm{i} B(B-\Omega)^{2}} \mathrm{e}^{-\mathrm{i} t \Omega}\left(|A|^{2} \bar{A}\right)^{\prime} \mathbf{P}_{\mathrm{c}} \varphi^{3} \\
& -\frac{3}{2} \lambda \frac{\mathrm{e}^{-\mathrm{i} B t}}{B(B-\Omega)}\left[\left|A_{0}\right|^{2} \bar{A}_{0}+\left.\left(|A|^{2} \bar{A}\right)^{\prime}\right|_{t=0}\right] \mathbf{P}_{\mathrm{c}} \varphi^{3} \\
& +\frac{3 \lambda}{2 \mathrm{i} B(B-\Omega)^{2}} \mathrm{e}^{\mathrm{i} B t} \int_{0}^{t} \mathrm{e}^{\mathrm{i} s(B-\Omega)}\left(|A|^{2} \bar{A}\right)^{\prime \prime} d s \mathbf{P}_{\mathrm{c}} \varphi^{3} \\
\eta_{27}^{n r}= & \frac{\lambda \bar{A}^{3}(t) \mathrm{e}^{-3 \mathrm{i} t \Omega}}{2 B(B-3 \Omega-\mathrm{i} 0)} \mathbf{P}_{\mathrm{c}} \varphi^{3}-\frac{3 \lambda}{2 \mathrm{i} B(B-3 \Omega-\mathrm{i} 0)^{2}} \mathrm{e}^{-3 \mathrm{i} t \Omega}\left(\bar{A}^{-3}\right)^{\prime} \mathbf{P}_{\mathrm{c}} \varphi^{3} \\
& -\frac{3}{2} \lambda \frac{\mathrm{e}^{-\mathrm{i} B t}}{B(B-3 \Omega-\mathrm{i} 0)}\left[\bar{A}_{0}^{3}+\left(\bar{A}^{3}\right)_{t=0}^{\prime}\right] \mathbf{P}_{\mathrm{c}} \varphi^{3} \\
& +\frac{3 \lambda}{2 \mathrm{i} B(B-3 \Omega-\mathrm{i} 0)^{2}} \mathrm{e}^{-\mathrm{i} B t} \int_{0}^{t} \mathrm{e}^{\mathrm{i} s(B-3 \Omega)}\left(\bar{A}^{3}\right)^{\prime \prime} d s \mathbf{P}_{\mathrm{c}} \varphi^{3}
\end{aligned}
$$

Recall that our goal is to elucidate the structure of the amplitude equation: $A^{\prime}=(2 \mathrm{i} \Omega)^{-1} \mathrm{e}^{-\mathrm{i} \Omega t} F$, in (4.17), where we first focused on the contribution: $(2 \mathrm{i} \Omega)^{-1} \mathrm{e}^{-\mathrm{i} \Omega t} F_{2}$. From (4.25) and Proposition 4.4. we see now that we need to obtain convenient expressions for

$$
\begin{aligned}
(2 \mathrm{i} \Omega)^{-1} \mathrm{e}^{-\mathrm{i} \Omega t} F_{2}\left(a, \eta_{2}^{n r}\right) & =\sum_{j=1}^{7}(2 \mathrm{i} \Omega)^{-1} \mathrm{e}^{-\mathrm{i} \Omega t} F_{2}\left(a, \eta_{2 j}^{n r}\right) \\
& =3 \lambda(2 \mathrm{i} \Omega)^{-1} a^{2} \mathrm{e}^{-\mathrm{i} \Omega t} \sum_{j=1}^{7} \int \varphi^{3} \eta_{2 j}^{n r} .
\end{aligned}
$$

Each of the seven terms contributing to $\eta_{2}^{n r}$ is expressed as a part which is $\mathcal{O}\left(|A|^{5}\right)$ plus an error term which is estimated in magnitude in section 5. The $\mathcal{O}\left(|A|^{5}\right)$ part and error terms are displayed in the following two propositions.

Proposition 4.6. Let

$$
\rho(\zeta) \equiv\left(\mathbf{P}_{\mathrm{c}} \varphi^{3}, B^{-1}(B-\zeta)^{-1} \mathbf{P}_{\mathrm{c}} \varphi^{3}\right)
$$

Then, 


$$
\begin{aligned}
& (2 \mathrm{i} \Omega)^{-1} \mathrm{e}^{-\mathrm{i} \Omega t} F_{2}\left(a, \eta_{21}^{n r}\right) \\
& \quad=\frac{\lambda^{2}}{\Omega} \rho(\Omega)\left[\frac{3}{4 \mathrm{i}}|A|^{2} A^{3} \mathrm{e}^{2 \mathrm{i} \Omega t}+\frac{3}{2 \mathrm{i}}|A|^{4} A+\frac{3}{4 \mathrm{i}}|A|^{4} \bar{A} \mathrm{e}^{-2 \mathrm{i} \Omega t}\right]+E_{21}^{n r} \\
& (2 \mathrm{i} \Omega)^{-1} \mathrm{e}^{-\mathrm{i} \Omega t} F_{2}\left(a, \eta_{22}^{n r}\right) \\
& \quad=\frac{\lambda^{2}}{\Omega} \rho(-\Omega)\left[\frac{9}{4 \mathrm{i}}|A|^{4} A+\frac{9}{2 \mathrm{i}}|A|^{4} \bar{A} \mathrm{e}^{-2 \mathrm{i} \Omega t}+\frac{9}{4 \mathrm{i}}|A|^{2} \bar{A}^{3} \mathrm{e}^{-4 \mathrm{i} \Omega t}\right]+E_{22}^{n r}
\end{aligned}
$$

$$
\begin{aligned}
& (2 \mathrm{i} \Omega)^{-1} \mathrm{e}^{-\mathrm{i} \Omega t} F_{2}\left(a, \eta_{23}^{n r}\right) \\
& =\frac{\lambda^{2}}{\Omega} \rho(-3 \Omega)\left[\frac{3}{4 \mathrm{i}}|A|^{4} \bar{A} \mathrm{e}^{-2 \mathrm{i} \Omega t}+\frac{3}{2}|A|^{2} \bar{A}^{3} \mathrm{e}^{-4 \mathrm{i} \Omega t}+\frac{3}{4} \bar{A}^{5} \mathrm{e}^{-6 \mathrm{i} \Omega t}\right]+E_{23}^{n r} \\
& (2 \mathrm{i} \Omega)^{-1} \mathrm{e}^{-\mathrm{i} \Omega t} F_{2}\left(a, \eta_{24}^{n r}\right) \\
& =\frac{\lambda^{2}}{\Omega} \rho(-3 \Omega)\left[\frac{3}{4} A^{5} \mathrm{e}^{4 \mathrm{i} \Omega t}+\frac{3}{4} A^{3} A^{2} \mathrm{e}^{2 \mathrm{i} \Omega t}+\frac{3}{4}|A|^{4} A\right]+E_{24}^{n r}
\end{aligned}
$$

$$
\begin{aligned}
& (2 \mathrm{i} \Omega)^{-1} \mathrm{e}^{-\mathrm{i} \Omega t} F_{2}\left(a, \eta_{25}^{n r}\right)= \\
& \frac{\lambda^{2}}{\Omega} \rho(-\Omega)\left[\frac{9}{4 \mathrm{i}}|A|^{2} A^{3} \mathrm{e}^{2 \mathrm{i} \Omega t}+\frac{9}{2 \mathrm{i}}|A|^{4} A+\frac{9}{4 \mathrm{i}}|A|^{4} A+\frac{9}{4 \mathrm{i}}|A|^{4} \bar{A} \mathrm{e}^{-2 \mathrm{i} \Omega t}\right]+E_{25}^{n r}
\end{aligned}
$$

$$
\begin{aligned}
& (2 \mathrm{i} \Omega)^{-1} \mathrm{e}^{-\mathrm{i} \Omega t} F_{2}\left(a, \eta_{26}^{n r}\right) \\
& =\frac{\lambda^{2}}{\Omega} \rho(\Omega)\left[\frac{9}{4 \mathrm{i}}|A|^{4} A+\frac{9}{2 \mathrm{i}}|A|^{4} \bar{A} \mathrm{e}^{-2 \mathrm{i} \Omega t}+\frac{9}{4 \mathrm{i}}|A|^{2} \bar{A}^{3} \mathrm{e}^{-4 \mathrm{i} \Omega t}\right]+E_{26}^{n r} \quad(4.45) \\
& (2 \mathrm{i} \Omega)^{-1} \mathrm{e}^{-\mathrm{i} \Omega t} F_{2}\left(a, \eta_{27}^{n r}\right) \\
& =\frac{\lambda^{2}}{\Omega} \rho(3 \Omega+\mathrm{i} 0)\left[\frac{3}{4 \mathrm{i}}|A|^{4} \bar{A} \mathrm{e}^{-2 \mathrm{i} \Omega t}+\frac{3}{2 \mathrm{i}}|A|^{2} \bar{A}^{3} \mathrm{e}^{-4 \mathrm{i} \Omega t}+\bar{A}^{-5} \mathrm{e}^{-6 \mathrm{i} \Omega t}\right]+E_{27}^{n r}
\end{aligned}
$$

In our analysis of the large time behavior $(t \rightarrow \pm \infty)$, we shall require an upper bound of the error terms $E_{2 j}^{n r}$ given in: 


\section{Proposition 4.7.}

$$
\begin{aligned}
& \left|E_{2 j}^{n r}\right| \leq C_{\varphi}|\lambda|^{2}|A|^{2}\left\{\left|A^{2}\right|\left|A^{\prime}\right|+\left\|\langle x\rangle^{-\sigma}\left(B-\zeta_{j}\right)^{-1} \mathrm{e}^{\mathrm{i} B t} \mathbf{P}_{\mathrm{c}} \varphi^{3}\right\|_{2}\right. \\
& \left.+\left\|\langle x\rangle^{-\sigma}\left(B-\zeta_{j}\right)^{-2} \int_{0}^{t} \mathrm{e}^{\mathrm{i} B(t-s)} \mathcal{O}\left(\left(|A|^{3}\right)^{\prime \prime}\right) d s \mathbf{P}_{\mathrm{c}} \varphi^{3}\right\|_{2}\right\},
\end{aligned}
$$

where $\zeta_{1}=\zeta_{6}=\Omega, \zeta_{2}=\zeta_{5}=-\Omega . \zeta_{3}=\zeta_{4}=-3 \Omega$, and $\zeta_{7}=3 \Omega+\mathrm{i} 0$.

In section 7, we shall estimate this expression using the decay estimates of section 2, in particular Proposition 2.2.

The desired form of the $A$-equation is now emerging. Use of Propositions 4.4 and 4.6 in (4.17) yields:

Proposition 4.8. The amplitude $A(t)$ satisfies the equation (see Proposition 4.6 for the definition of $\rho(\zeta))$ :

$$
\begin{aligned}
A^{\prime}= & \frac{-\mathrm{i} \lambda}{2 \Omega}\|\varphi\|_{4}^{4}\left(3|A|^{2} A+A^{3} \mathrm{e}^{2 \mathrm{i} \Omega t}+3|A|^{2} \bar{A} \mathrm{e}^{-2 \mathrm{i} \Omega t}+\vec{A}^{3} \mathrm{e}^{-4 \mathrm{i} \Omega t}\right) \\
& -\frac{3}{4} \frac{\lambda^{2}}{\Omega} \Gamma|A|^{4} A-\frac{3 \mathrm{i}}{4 \Omega} \lambda^{2}[\Lambda-5 \rho(\Omega)+3 \rho(-\Omega)+\rho(-3 \Omega)]|A|^{4} A \\
& -\frac{3 \lambda^{2}}{4 \Omega} A^{5} \mathrm{e}^{4 \mathrm{i} \Omega t}[\mathrm{i} \Lambda+\Gamma-\rho(-3 \Omega)] \\
& -\frac{3 \mathrm{i} \lambda^{2}}{4 \Omega}|A|^{2} A^{3} \mathrm{e}^{2 \mathrm{i} \Omega t}[-2 \Lambda+2 i \Gamma+\rho(\Omega)+\mathrm{i} \rho(-3 \Omega)+3 \rho(-\Omega)] \\
& -\frac{3 \mathrm{i} \lambda^{2}}{4 \Omega}|A|^{4} \bar{A} \mathrm{e}^{-2 \mathrm{i} \Omega t}[7 \rho(\Omega)+9 \rho(-\Omega)+\rho(-3 \Omega)+\rho(3 \Omega+\mathrm{i} 0)] \\
& -\frac{3 \mathrm{i} \lambda^{2}}{4 \Omega}|A|^{2} \bar{A}^{3} \mathrm{e}^{-4 \mathrm{i} \Omega t}[3 \rho(-\Omega)-2 \mathrm{i} \rho(-3 \Omega)+\rho(3 \Omega+\mathrm{i} 0)+3 \rho(\Omega)] \\
& -\frac{3 \mathrm{i} \lambda^{2}}{4 \Omega} \bar{A}^{5} \mathrm{e}^{-6 \mathrm{i} \Omega t}\left[\mathrm{i} \rho(-3 \Omega)-\frac{4}{3} \mathrm{i} \rho(3 \Omega+\mathrm{i} 0)\right]+\mathbf{E}
\end{aligned}
$$

where 


$$
\begin{aligned}
\mathbf{E}= & (2 \mathrm{i} \Omega)^{-1} \mathrm{e}^{\mathrm{i} \Omega t} 3 \lambda a \int \varphi \eta^{2} \\
& +(2 \mathrm{i} \Omega)^{-1} \lambda \mathrm{e}^{-\mathrm{i} \Omega t} \int \varphi \eta^{3}+\sum_{j=1}^{7} E_{2 j}^{n r} \\
& +(2 \mathrm{i} \Omega)^{-1} \mathrm{e}^{-\mathrm{i} \Omega t}\left[F_{2}\left(a, \eta_{1}\right)+F_{2}\left(a, \eta_{3}\right)\right] \\
& +F_{2}\left(a, \eta_{*}^{n r 1}+\eta_{*}^{n r 2}\right)
\end{aligned}
$$

Here, $F_{2}\left(a, \eta_{j}\right)$ is given by (4.18).

In the next section we show how to rewrite (4.48) in a manner which makes explicit which terms determine the large time behavior of the amplitude and phase of $A(t)$.

\section{Dispersive Hamiltonian normal form}

To analyze the asymptotic behavoir of $A(t)$ (or equivalently $a(t)$ ) and $\eta(t, x)$ as $t \rightarrow \infty$ it is useful to use the idea of normal forms [3], [26], [52] from dynamical systems theory. We derive a perturbed normal form which makes the anticipated large time behavior of solutions transparent.

Proposition 5.1. There exists a smooth near-identity change of variables, $A \mapsto \tilde{A}$ with the following properties:

$$
\begin{aligned}
\tilde{A} & =A+h(A, t) \\
h(A, t) & =O\left(|A|^{3}\right), \quad|A| \rightarrow 0 \\
h(A, t) & =h\left(A, t+2 \pi \Omega^{-1}\right),
\end{aligned}
$$

and such that in terms of $\tilde{A}$ equation (4.48) becomes:

$$
\begin{aligned}
\tilde{A}^{\prime}= & \mathrm{i} \lambda c_{21}|\tilde{A}|^{2} \tilde{A}+\lambda^{2} d_{32}|\tilde{A}|^{4} \tilde{A}+\mathrm{i} \lambda^{2} c_{32}|\tilde{A}|^{4} \tilde{A} \\
& +O\left(|\tilde{A}|^{7}\right)+\tilde{\mathbf{E}},
\end{aligned}
$$

where $\lambda^{2} d_{32}=-\frac{3}{4} \frac{\lambda^{2}}{\Omega} \Gamma<0$. The constants $c_{21}$ and $c_{32}$ are real numbers, explicitly calculable in terms of the coefficients appearing in (4.48). The remainder term $|\tilde{\mathbf{E}}|$ is estimable in terms of $|\mathbf{E}|$ for $|\tilde{A}|<1$ (equivalently $|A|<1)$. 
Remarks. The point of this proposition is that in the new variables the dynamics evidently have a dissipative aspect. Specifically, neglecting the perturbation to the normal form one has:

$$
\partial_{t}|\tilde{A}|^{2}=-\frac{3}{8} \frac{\lambda^{2}}{\Omega} \Gamma|\tilde{A}|^{6}<0
$$

We therefore refer to (5.2) as a dispersive Hamiltonian normal form. In finite dimensional Hamiltonian systems, the normal form associated with a one degree of freedom Hamiltonian system is an equation like (5.2), but with all the coefficients of the terms $|A|^{2 m} A$ being purely imaginary. Here we find that resonant coupling to an infinite dimensional dispersive wave field can lead to a normal form with general complex coefficients, which in our context implies the internal damping effect described above. See section 8 for further discussion.

We now present an elementary derivation of the change of variables (5.1) leading to (5.2). Equation (4.48) is of the form:

$$
A^{\prime}=\sum_{j \in\{3,5\}} \sum_{k+l=j} \alpha_{k l} A^{k} \bar{A}^{l} \mathrm{e}^{\mathrm{i}(k-l-1) \Omega t}+\mathbf{E}
$$

where the coefficients $\alpha_{k l}$ can be read off (4.48). The proof we present is quite general and shows, in particular, that the normal form for equations like (5.4) is (5.2).

Proposition 5.2. There is a change of variables, as in (5.1), such that equation (5.4) is mapped to:

$$
\begin{aligned}
\tilde{A}^{\prime}= & k_{21}|\tilde{A}|^{2} \tilde{A}+k_{32}|\tilde{A}|^{4} \tilde{A} \\
& +\mathcal{O}\left(|\tilde{A}|^{7}\right)+\tilde{\mathbf{E}}
\end{aligned}
$$

where

$$
\begin{gathered}
\tilde{\mathbf{E}}=\mathbf{E} \circ(I+h) \\
k_{21}=\alpha_{21} \\
k_{32}=\alpha_{32}+(2 \mathrm{i} \Omega)^{-1}\left[\frac{3}{2}\left|\alpha_{03}\right|^{2}-2 \alpha_{30} \alpha_{12}+2\left|\alpha_{12}\right|^{2}\right]
\end{gathered}
$$

The conclusion in Proposition 5.1 concerning the "damping coefficient" $d_{32}$ depends on the particular properties of the coefficients in (4.48). In particular we have from (4.48) and (5.4) that: 


$$
\begin{aligned}
& \alpha_{21}=\alpha_{12}=\frac{3 \lambda}{2 \mathrm{i} \Omega}\|\varphi\|_{4}^{4}, \\
& \alpha_{03}=\frac{\lambda}{2 \mathrm{i} \Omega}\|\varphi\|_{4}^{4}, \\
& \alpha_{32}=-\frac{3 \lambda^{2}}{4 \Omega} \Gamma-\frac{3 \mathrm{i} \lambda^{2}}{4 \Omega}[\Lambda-5 \rho(\Omega)+3 \rho(-\Omega)+\rho(-3 \Omega)] .
\end{aligned}
$$

From these formulae, we have $\lambda^{2} d_{32}$ is given by the real part of $\alpha_{32}$, the "singular" Fermi golden rule contribution.

Remark. The construction of the map $A \mapsto \tilde{A}$ can be applied as well to equations of the form (5.4) where the right hand side is an arbitrary expansion in powers of $A$ and $\bar{A}$.

To make the structure clear we write out the equation with a particular ordering of terms:

$$
A^{\prime}=\alpha_{21}|A|^{2} A+\alpha_{32}|A|^{4} A+O_{3}(A)+O_{5}(A)+\mathbf{E},
$$

where

$$
\begin{aligned}
O_{3}(A)= & \alpha_{30} A^{3} \mathrm{e}^{2 \mathrm{i} \Omega t}+\alpha_{12} A \vec{A}^{2} \mathrm{e}^{-2 \mathrm{i} \Omega t}+\alpha_{03} \bar{A}^{3} \mathrm{e}^{-4 \mathrm{i} \Omega t}, \quad \text { and } \\
O_{5}(A)= & \alpha_{50} A^{5} \mathrm{e}^{4 \mathrm{i} \Omega t}+\alpha_{41} A^{4} \bar{A} \mathrm{e}^{2 \mathrm{i} \Omega t}+\alpha_{23} A^{2} \bar{A}^{3} \mathrm{e}^{-2 \mathrm{i} \Omega t} \\
& +\alpha_{14} A \bar{A}^{4} e^{-4 \mathrm{i} \Omega t}+\alpha_{05} \bar{A}^{5} \mathrm{e}^{-6 \mathrm{i} \Omega t}
\end{aligned}
$$

Note that each term in $O_{3}(A)$ and $O_{5}(A)$ is of the form: oscillatory function of $t$ times order $\mathcal{O}\left(|A|^{3}\right)$ or $\mathcal{O}\left(|A|^{5}\right)$.

The computations that follow, though elementary, are rather lengthy so we first outline the strategy of our proof. Integration of (5.8) gives

$$
A=A_{0}+\int_{0}^{t} \alpha_{21}|A|^{2} A+\alpha_{32}|A|^{4} A+O_{3}(A)+O_{5}(A)+\mathbf{E} d s
$$

The idea is that terms with explicit periodic oscillations average to zero and can be neglected in determining the large time behavior of the solution. Our strategy is now to expand the explicitly oscillatory terms using repeated integrations by parts and to make explicit all terms up to and including order $|A|^{5}$. The computation has two stages. In stage one, after repeated integration by parts the equation (5.11) is expressed in the equivalent form: 


$$
\begin{aligned}
A(t)-H_{1}(A(t), t)= & A_{0}-H_{1}\left(A_{0}, 0\right) \\
& +\int_{0}^{t} \text { "resonant" terms like }|A|^{2} A,|A|^{4} A d s \\
& +\int_{0}^{t} \text { terms of type } O_{5}(A) d s \\
& + \text { higher order corrections }
\end{aligned}
$$

This suggests the change of variables $A \mapsto A_{1}=A(t)-H_{1}(A(t), t)$, giving

$$
\begin{aligned}
A_{1}(t)= & A_{10}+\int_{0}^{t} \text { terms like }\left|A_{1}\right|^{2} A_{1},\left|A_{1}\right|^{4} A_{1} d s \\
& + \text { terms of type } O_{5}\left(A_{1}\right) \\
& + \text { higher order corrections. }
\end{aligned}
$$

The latter equation is equivalent to a differential equation of the form:

$$
\begin{aligned}
A_{1}^{\prime}(t)= & \text { terms like }\left|A_{1}\right|^{2} A_{1},\left|A_{1}\right|^{4} A_{1} \\
& + \text { terms of type } O_{5}\left(A_{1}\right) \\
& + \text { higher order corrections. }
\end{aligned}
$$

which is a step closer to the form of the equation we seek. A second iteration of this process yields the result.

We now embark on the details of the proof.

Expansion of $\int_{0}^{t} O_{3}(A) d s$ :

Integration of the expression in (5.9) gives

$$
\begin{aligned}
\int_{0}^{t} O_{3}(A) d s= & h_{3}(A(t), t)-h_{3}\left(A_{0}, 0\right) \\
& -\frac{3 \alpha_{30}}{2 i \Omega} \int_{0}^{t} \mathrm{e}^{2 \mathrm{i} \Omega s} A^{2} A^{\prime} d s+\frac{\alpha_{12}}{2 i \Omega} \int_{0}^{t} \mathrm{e}^{-2 \mathrm{i} \Omega t}\left(A \overrightarrow{A^{2}}\right)^{\prime} d s \\
& +\frac{\alpha_{03}}{4 \mathrm{i} \Omega} \int_{0}^{t} \mathrm{e}^{-4 \mathrm{i} \Omega s}\left(\overrightarrow{A^{3}}\right)^{\prime} d s,
\end{aligned}
$$


where

$$
h_{3}(A, t)=\frac{\alpha_{30}}{2 \mathrm{i} \Omega} A^{3} \mathrm{e}^{2 i \Omega t}-\frac{\alpha_{12}}{2 \mathrm{i} \Omega} A \vec{A}^{2} \mathrm{e}^{-2 \mathrm{i} \Omega t}-\frac{\alpha_{03}}{4 \mathrm{i} \Omega} \vec{A}^{3} \mathrm{e}^{-4 \mathrm{i} \Omega t}
$$

We now replace $A^{\prime}$ in (5.15) by its abbreviated expression given in (5.8). Thus we have:

$$
\begin{aligned}
\int_{0}^{t} O_{3}(A) d s= & h_{3}(A(t), t)-h_{3}\left(A_{0}, 0\right) \\
& -\frac{3 \alpha_{30}}{2 \mathrm{i} \Omega} \int_{0}^{t} \mathrm{e}^{2 \mathrm{i} \Omega s} A^{2}\left[\alpha_{21}|A|^{2} A+O_{3}(A)+\mathcal{O}\left(|A|^{5}\right)+\mathbf{E}\right] d s \\
& +\frac{\alpha_{12}}{2 \mathrm{i} \Omega} \int_{0}^{t} \mathrm{e}^{-2 \mathrm{i} \Omega s} \bar{A}^{2}\left[\alpha_{21}|A|^{2} A+O_{3}(A)+\mathcal{O}\left(|A|^{5}\right)+\mathbf{E}\right] d s \\
& +\frac{\alpha_{12}}{\mathrm{i} \Omega} \int_{0}^{t} \mathrm{e}^{-2 \mathrm{i} \Omega s}|A|^{2}\left[\bar{\alpha}_{21}|A|^{2} \bar{A}+\bar{O}_{3}(A)+\mathcal{O}\left(|A|^{5}\right)+\overline{\mathbf{E}}\right] d s \\
& +3 \frac{\alpha_{03}}{4 \mathrm{i} \Omega} \int_{0}^{t} \mathrm{e}^{-4 \mathrm{i} \Omega s} \bar{A}^{2}\left[\bar{\alpha}_{21}|A|^{2} \bar{A}+\bar{O}_{3}(A)+\mathcal{O}\left(|A|^{5}\right)+\overline{\mathbf{E}}\right] d s
\end{aligned}
$$

Substitution of (5.9) into (5.17) and integrating by parts, we arrive at the following expression:

$$
\begin{aligned}
\int_{0}^{t} O_{3}(A) d s= & h_{3}(A(t), t)+h_{3 a}(A(t), t)-h_{3}\left(A_{0}, 0\right)-h_{3 a}\left(A_{0}, 0\right) \\
& +\frac{1}{2 \mathrm{i} \Omega}\left(\frac{3}{2}\left|\alpha_{03}\right|^{2}-2 \alpha_{30} \alpha_{12}+2\left|\alpha_{12}\right|^{2}\right) \int_{0}^{t}|A|^{4} A d s \\
& +\int_{0}^{t} \mathcal{O}\left(|A|^{2}\left(|A|^{5}+|\mathbf{E}|\right)\right) d s,
\end{aligned}
$$

where 


$$
\begin{aligned}
h_{3 a}(A, t)= & {\left[-\frac{\alpha_{12} \bar{\alpha}_{03}}{2 \Omega^{2}}+\frac{3 \alpha_{30} \alpha_{21}}{4 \Omega^{2}}\right] A^{4} \bar{A}^{2 \mathrm{i} \Omega t}-\frac{3 \alpha_{30}^{2}}{8 \Omega^{2}} A^{5} \mathrm{e}^{4 \mathrm{i} \Omega t} } \\
& +\frac{1}{4 \Omega^{2}}\left(\alpha_{21} \alpha_{12}+\frac{3}{2} \alpha_{03} \bar{\alpha}_{12}-3 \alpha_{30} \alpha_{03}+2 \alpha_{12} \bar{\alpha}_{21}\right) A^{2} \bar{A}^{3} \mathrm{e}^{-2 \mathrm{i} \Omega t} \\
& +\frac{1}{8 \Omega^{2}}\left(\alpha_{12}^{2}+\frac{3}{2} \alpha_{03} \bar{\alpha}_{21}+2 \alpha_{12} \bar{\alpha}_{30}\right) A \bar{A}^{4} \mathrm{e}^{-4 \mathrm{i} \Omega t} \\
& +\frac{1}{4 \Omega^{2}}\left(\frac{1}{3} \alpha_{03} \alpha_{12}+\frac{1}{2} \alpha_{03} \bar{\alpha}_{30}\right) \bar{A}^{5} \mathrm{e}^{-6 \mathrm{i} \Omega t}
\end{aligned}
$$

Referring back to (5.8), we see we must now obtain an Expansion of $\int_{0}^{t} O_{5}(A) d s$ :

From (5.10) we have, after integration by parts:

$$
\begin{aligned}
\int_{0}^{t} O_{5}(A) d s= & h_{3 b}(A(t), t)-h_{3 b}\left(A_{0}, 0\right) \\
& +\int_{0}^{t} \mathcal{O}\left(|A|^{4}\left(|A|^{3}+|\mathbf{E}|\right)\right) d s,
\end{aligned}
$$

where

$$
\begin{aligned}
h_{3 b}(A, t)= & \frac{\alpha_{50}}{4 \mathrm{i} \Omega} A^{5} \mathrm{e}^{4 \mathrm{i} \Omega t}+\frac{\alpha_{41}}{2 \mathrm{i} \Omega} A^{4} \bar{A} \mathrm{e}^{2 \mathrm{i} \Omega t}-\frac{\alpha_{23}}{2 \mathrm{i} \Omega} A^{2} \bar{A}^{3} \mathrm{e}^{-2 \mathrm{i} \Omega t} \\
& -\frac{\alpha_{14}}{4 \mathrm{i} \Omega} A \bar{A}^{4} \mathrm{e}^{-4 \mathrm{i} \Omega t}-\frac{\alpha_{05}}{6 \mathrm{i} \Omega} \bar{A}^{5} \mathrm{e}^{-6 \mathrm{i} \Omega t} .
\end{aligned}
$$

Therefore from (5.11) and our computations we have:

$$
\begin{aligned}
& A-h_{3}(A, t)-h_{3 a}(A, t)-h_{3 b}(A, t) \\
& =A_{0}-h_{3}\left(A_{0}, 0\right)-h_{3 a}\left(A_{0}, 0\right)-h_{3 b}\left(A_{0}, 0\right) \\
& \quad+\int_{0}^{t} \alpha_{21}|A|^{2} A+\alpha_{32}|A|^{4} A d s \\
& \quad+\frac{1}{2 \mathrm{i} \Omega}\left(\frac{3}{2}\left|\alpha_{03}\right|^{2}-2 \alpha_{30} \alpha_{12}+2\left|\alpha_{12}\right|^{2}\right) \int_{0}^{t}|A|^{4} A d s+\int_{0}^{t} \mathbf{E} d s
\end{aligned}
$$




$$
+\int_{0}^{t} \mathcal{O}\left(|A|^{4}\left(|A|^{3}+|\mathbf{E}|\right)\right)+\mathcal{O}\left(|A|^{2}\left(|A|^{5}+|\mathbf{E}|\right)\right) d s .
$$

This suggests the change of variables:

$$
\begin{aligned}
A_{1} & \equiv A-H_{1}(A, t), \quad \text { where } \\
H_{1}(A, t) & =h_{3}(A, t)+h_{3 a}(A, t)+h_{3 b}(A, t),
\end{aligned}
$$

which for small $|A|$, is a near-identity change of variables. Using this change of variables we have that (5.22) becomes

$$
\begin{aligned}
A_{1}= & A_{10}+\int_{0}^{t} \alpha_{21}\left|A_{1}\right|^{2} A_{1}+\alpha_{32}\left|A_{1}\right|^{4} A_{1} d s \\
& +\frac{1}{2 \mathrm{i} \Omega}\left(\frac{3}{2}\left|\alpha_{03}\right|^{2}-2 \alpha_{30} \alpha_{12}+2\left|\alpha_{12}\right|^{2}\right) \int_{0}^{t}\left|A_{1}\right|^{4} A_{1} d s \\
& +\int_{0}^{t}\left(2 \alpha_{21}\left|A_{1}\right|^{2} H_{1}\left(A_{1}, s\right)+A_{1}^{2} \bar{H}_{1}\left(A_{1}, s\right)\right) d s+\int_{0}^{t} \mathbf{E}_{\mathbf{1}} d s \\
& +\int_{0}^{t} \mathcal{O}\left(\left|A_{1}\right|^{4}\left(\left|A_{1}\right|^{3}+\left|\mathbf{E}_{1}\right|\right)\right)+\mathcal{O}\left(\left|A_{1}\right|^{2}\left(\left|A_{1}\right|^{5}+\left|\mathbf{E}_{1}\right|\right)\right) d s .
\end{aligned}
$$

We expand the terms involving $H_{1}(A, t)$. Using integration by parts, as above, we obtain:

$$
\begin{aligned}
2 \alpha_{21} \int_{0}^{t}\left|A_{1}\right|^{2} H_{1} d s= & h_{5 a}\left(A_{1}, t\right)-h_{5 a}\left(A_{10}, 0\right) \\
& +\int_{0}^{t} \mathcal{O}\left(\left|A_{1}\right|^{4}\left(\left|A_{1}\right|^{3}+\left|\mathbf{E}_{\mathbf{1}}\right|\right)\right) d s
\end{aligned}
$$

and

$$
\begin{aligned}
\alpha_{21} \int_{0}^{t} A_{1}^{2} \bar{H}_{1} d s= & h_{5 b}\left(A_{1}, t\right)-h_{5 b}\left(A_{10}, 0\right) \\
& +\int_{0}^{t} \mathcal{O}\left(\left|A_{1}\right|^{4}\left(\left|A_{1}\right|^{3}+\left|\mathbf{E}_{\mathbf{1}}\right|\right)\right) d s,
\end{aligned}
$$


where

$$
\begin{aligned}
h_{5 a}(A, t)= & -\frac{\alpha_{21} \alpha_{30}}{2 \Omega^{2}} \mathrm{e}^{2 \mathrm{i} \Omega t} A_{1}^{4} \bar{A}_{1}-\frac{\alpha_{21} \alpha_{12}}{2 \Omega^{2}} \mathrm{e}^{-2 \mathrm{i} \Omega t} A_{1}^{2} \bar{A}_{1}^{3} \\
& -\frac{\alpha_{21} \alpha_{03}}{8 \Omega^{2}} \mathrm{e}^{-4 \mathrm{i} \Omega t} A_{1} \bar{A}_{1}^{4} \\
h_{5 b}(A, t)= & -\frac{\alpha_{21} \bar{\alpha}_{30}}{4 \Omega^{2}} \mathrm{e}^{-2 \mathrm{i} \Omega t} A_{1}^{2} \bar{A}_{1}^{3}-\frac{\alpha_{21} \bar{\alpha}_{12}}{4 \Omega^{2}} \mathrm{e}^{2 \mathrm{i} \Omega t} A_{1}^{4} \bar{A}_{1} \\
& -\frac{\alpha_{21} \bar{\alpha}_{03}}{16 \Omega^{2}} \mathrm{e}^{4 \mathrm{i} \Omega t} A_{1}^{5} \\
H_{5}(A, t)= & h_{5 a}(A, t)+h_{5 b}(A, t)
\end{aligned}
$$

Thus

$$
\begin{aligned}
A_{1}-H_{5}\left(A_{1}(t), t\right)= & A_{10}-H_{5}\left(A_{10}, 0\right) \\
& +\int_{0}^{t} \alpha_{21}\left|A_{1}\right|^{2} A_{1}+\alpha_{32}\left|A_{1}\right|^{4} A_{1} d s \\
& +\frac{1}{2 \mathrm{i} \Omega}\left(\frac{3}{2}\left|\alpha_{03}\right|^{2}-2 \alpha_{30} \alpha_{12}+2\left|\alpha_{12}\right|^{2}\right) \int_{0}^{t}\left|A_{1}\right|^{4} A_{1} d s \\
& +\int_{0}^{t} \mathcal{O}\left(\left|A_{1}\right|^{7}+\left|A_{1}\right|^{2}\left|\mathbf{E}_{1}\right|+\left|\mathbf{E}_{1}\right|\right) d s
\end{aligned}
$$

Now define

$$
\tilde{A} \equiv A_{1}-H_{5}\left(A_{1}, t\right) .
$$

In terms of this new variable we have:

$$
\begin{aligned}
\tilde{A}=\tilde{A}_{0} & +\int_{0}^{t} \alpha_{21}|\tilde{A}|^{2} \tilde{A}+\alpha_{32}|\tilde{A}|^{4} \tilde{A} d s \\
& +\frac{1}{2 \mathrm{i} \Omega}\left(\frac{3}{2}\left|\alpha_{03}\right|^{2}-2 \alpha_{30} \alpha_{12}+2\left|\alpha_{12}\right|^{2}\right) \int_{0}^{t}|\tilde{A}|^{4} \tilde{A} d s \\
& +\int_{0}^{t} \mathcal{O}\left(|\tilde{A}|^{7}+|\tilde{\mathbf{E}}|\right) d s
\end{aligned}
$$


where $|\tilde{\mathbf{E}}|$ is estimable in terms of $|\mathbf{E}|$ for $|A|<1$. This completes the proof.

\section{Asymptotic behavior of solutions of perturbed normal form equations}

We now consider the large time behavior of solutions to the ordinary differential equations of the form (5.2). In particular, we compare the behavior of solutions of (5.2) to those of the equations with $\tilde{\mathbf{E}}$ set equal to zero.

Thus we consider the equations:

$$
\begin{aligned}
& \beta^{\prime}=\mathrm{i} c_{21}|\beta|^{2} \beta+\left(\mathrm{i} c_{32}-\gamma\right)|\beta|^{4} \beta+\mathbf{Q}(t), \\
& \alpha^{\prime}=\mathrm{i} c_{21}|\alpha|^{2} \alpha+\left(\mathrm{i} c_{32}-\gamma\right)|\alpha|^{4} \alpha
\end{aligned}
$$

wherer $\gamma>0$. We first consider the uperturbed equation, (6.2). Multiplication of (6.2) by $\bar{\alpha}$ and taking the real part of the resulting equation yields the equation:

$$
r^{\prime}=-2 \gamma r^{3}, \quad r=|\alpha|^{2} .
$$

Integration of (6.3) yields:

$$
r^{2}(t)=r_{0}^{2}\left(1+4 \gamma r_{0}^{2} t\right)^{-1}
$$

To prove the above lemma, we begin by multiplying (6.1) by $\bar{\beta}$ and taking the real part of the resulting equation. This gives:

$$
r^{\prime}(t)=-2 \gamma r^{3}(t)+\mathbf{Q}(t) \bar{\beta}(t)+\overline{\mathbf{Q}}(t) \beta(t),
$$

which implies the differential inequality:

$$
r^{\prime}(t) \leq-2 \gamma r^{3}(t)+2|\mathbf{Q}(t)| r^{\frac{1}{2}}(t) .
$$

We now prove

Lemma 6.1. Suppose $r(t)=|\beta(t)|^{2}$ satisfies (6.6) with:

$$
|\mathbf{Q}(t)| \leq Q_{0}\langle t\rangle^{-\frac{5}{4}-\delta}, \quad \delta \geq 0 .
$$

Then, 


$$
|\beta(t)|^{4} \leq\left(1+4\left|\beta_{0}\right|^{4} \gamma t\right)^{-1}\left(2\left|\beta_{0}\right|^{4}+\frac{m_{*}^{2} Q_{0}^{\frac{8}{5}}}{\gamma^{\frac{3}{5}}\left\langle t t^{\frac{8}{\delta} \delta}\right.}\right),
$$

where $m_{*}=\max \left\{1,4 \gamma\left|\beta_{0}\right|^{4}\right\}$.

Proof. Use of (6.7) in (6.6) gives the inequality

$$
r^{\prime}(t) \leq-2 \gamma r^{3}(t)+2 Q_{0}\langle t\rangle^{-\frac{5}{4}-\delta} r^{\frac{1}{2}}(t) .
$$

Multiplication by $r(t)$ gives:

$$
z^{\prime}(t) \leq-4 \gamma z^{2}(t)+4 Q_{0}\langle t\rangle^{-\frac{5}{4}-\delta} z^{\frac{3}{4}}(t), \quad \text { where } z(t)=r^{2}(t) .
$$

Note that the equation

$$
\zeta^{\prime}(t)=-4 \gamma \zeta^{2}(t), \zeta(0)=z_{0}=\left|\beta_{0}\right|^{4}
$$

has solutions:

$$
\zeta(t)=z_{0}\left(1+4 z_{0} \gamma t\right)^{-1}
$$

Anticipating this as the dominant behavior for large $t$, we define:

$$
z(t) \equiv \zeta(t) R(t)
$$

Substitution into (6.10) and simplifying gives:

$$
R^{\prime}(t) \leq \frac{-4 \gamma z_{0}}{1+4 z_{0} \gamma t} R(R-1)+C \frac{Q_{0}}{\left|z_{0}\right|^{\frac{1}{4}}}\left(1+4 z_{0} \gamma t\right)^{\frac{1}{4}}\langle t\rangle^{-\frac{5}{4}-\delta} R^{\frac{3}{4}}(t) .
$$

We now consider the last term in (6.14). Noting that

$$
(1+t)^{-1} \leq m_{*}\left(1+4 \gamma z_{0} t\right)^{-1}, \quad m_{*}=\max \left\{1,4 \gamma z_{0}\right\},
$$

we have for any $\varepsilon>0$ :

$$
\begin{aligned}
C \frac{Q_{0}}{\left|z_{0}\right|^{\frac{1}{4}}} \frac{\left(1+4 z_{0} \gamma t\right)^{\frac{1}{4}}}{\langle t\rangle^{\frac{5}{4}+\delta}} R^{\frac{3}{4}}(t) & \leq C \frac{Q_{0}}{\left|z_{0}\right|^{\frac{1}{4}}\langle t\rangle^{\frac{1}{4}}} R^{\frac{3}{4}} \\
& \leq C \frac{Q_{0} m_{*}^{\frac{5}{8}}}{\varepsilon\left|z_{0}\right|^{\frac{1}{4}}\langle t\rangle^{\frac{5}{8}+\delta}} \times \frac{\varepsilon R^{\frac{3}{4}}(t)}{\left(1+4 z_{0} \gamma t\right)^{\frac{3}{8}}}
\end{aligned}
$$




$$
\leq C_{1} \frac{Q_{0}^{\frac{8}{5}} m_{*}^{\frac{3}{5}}}{\varepsilon^{\frac{8}{5}} z_{0}^{\frac{2}{5}}\langle t\rangle^{1+\frac{8}{5} \delta}}+C_{2} \frac{\frac{8}{\varepsilon^{3}} R^{2}(t)}{\left(1+4 z_{0} \gamma t\right)}
$$

The last inequality follows from the inequality: $a b \leq p^{-1}(\varepsilon a)^{p}+$ $q^{-1}(b / \varepsilon)^{q}, \quad p^{-1}+q^{-1}=1$, for the choice $p=\frac{8}{3}$ and $q=\frac{8}{5}$.

This last estimate can now be used in (6.14) and implies:

$$
R^{\prime} \leq \frac{-4 \gamma z_{0}+C_{2} \varepsilon^{\frac{8}{3}}}{1+4 z_{0} \gamma t} R^{2}(t)+\frac{4 \gamma z_{0}}{1+4 z_{0} \gamma t} R(t)+C_{1} \frac{Q_{0}^{\frac{8}{5}} m_{*}^{\frac{3}{5}}}{\varepsilon^{\frac{8}{5}} z_{0}^{\frac{2}{5}}\langle t\rangle^{1+\frac{8}{5} \delta}}
$$

which when setting $C_{2} \varepsilon^{\frac{8}{3}}=2 \gamma z_{0}$, is

$$
\leq-\frac{2 \gamma z_{0}}{1+4 z_{0} \gamma t} R(R-2)+C \frac{Q_{0}^{\frac{8}{5}} m_{*}}{\gamma^{\frac{3}{5} z_{0}}\langle t\rangle^{1+\frac{8}{3} \delta}} .
$$

Now set $R=2+S$. Since the term proportional to $S^{2}$ is negative, we obtain

$$
S^{\prime} \leq \frac{-4 \gamma z_{0}}{1+4 z_{0} \gamma t} S+C \frac{Q_{0}^{\frac{8}{5}} m_{*}}{\gamma^{\frac{3}{5}} z_{0}\langle t\rangle^{1+\frac{8}{5} \delta}}
$$

Multiplication by $1+4 z_{0} \gamma t$ yields:

$$
\begin{aligned}
\partial_{t}\left[\left(1+4 z_{0} \gamma t\right) S(t)\right] & \leq C \frac{Q_{0}^{\frac{8}{5}} m_{*}}{\gamma^{\frac{3}{5}} z_{0}} \frac{1+4 z_{0} \gamma t}{(1+t)} \frac{1}{\langle t\rangle^{\frac{8}{5} \delta}} \\
& \leq C \frac{Q_{0}^{\frac{8}{5}} m_{*}^{2}}{\gamma^{\frac{3}{5}} z_{0}} \frac{1}{\langle t\rangle^{\frac{8}{5} \delta}} .
\end{aligned}
$$

Integration of (6.18) from 0 to $t$ implies:

$$
S(t) \leq C \frac{Q_{0}^{\frac{8}{5}} m_{*}^{2}}{\gamma^{\frac{3}{5}} z_{0}} \frac{1}{\langle t\rangle^{\frac{8}{5} \delta}} .
$$

The Lemma now follows from (6.19) and the relation:

$$
|\beta(t)|^{4} \equiv z(t) \equiv \zeta(t) R(t) \equiv \zeta(t)(2+S(t))
$$




\section{Asymptotic behavior of solutions of the nonlinear Klein-Gordon equation}

In section 2 we proved that local in time solutions, $\mathbf{u}(\mathrm{t})$, exist in the space $C^{0}\left(-T^{*}, T_{*} ; \mathbf{X}_{\mathbf{0}}\right)$, for some $T_{*}, T^{*}>0$. We now study obtain the required ' $a$ priori' bounds to ensure (a) persistence of the solution, $\mathbf{u}(\mathrm{t})$, as a continuous function with values in $\mathbf{X}_{\mathbf{0}}\left(T^{*}=T_{*}=\infty\right)$ and (b) the decay of soltuions as $t \rightarrow \pm \infty$ in suitable norms. Due to the linear estimates of section 2, we require more stringent hypotheses on $u_{0}$ and $u_{1}$. These linear estimates require finiteness of $W^{1,4 / 3}$ and $W^{1,8 / 7}$ norms which, by interpolation, are controlled under the assumption $\mathbf{u}_{0} \in \mathbf{X}$ (see Section 2). Specifically, $u_{0} \in W^{2,2} \cap W^{2,1}$ and $u_{1} \in W^{1,2} \cap W^{1,1}$.

Using the results of the previous section, the original dynamical systems (4.1) can now be rewritten as:

$$
\begin{aligned}
u(x, t) & =a(t) \varphi(x)+\eta(x, t), \\
\eta(x, t) & =\eta_{1}(x, t)+\eta_{2}(x, t)+\eta_{3}(x, t), \\
a(t) & =A(t) \mathrm{e}^{\mathrm{i} \Omega t}+\bar{A}(t) \mathrm{e}^{-\mathrm{i} \Omega t}, \\
A(t) & =\tilde{A}(t)+h(\tilde{A}(t), t),
\end{aligned}
$$

Here, $h(\tilde{A}, t)$ is a smooth periodic function of $t$, cubic in $\tilde{A}$ for $\tilde{A} \rightarrow 0, \eta_{1}$ and $\eta_{2}$ are defined by (4.12)-(4.13), and $\tilde{A}(t)$ satisfied the perturbed normal form equation:

$$
\tilde{A}^{\prime}=\mathrm{i} \lambda c_{21}|\tilde{A}|^{2} \tilde{A}-\gamma|\tilde{A}|^{4} \tilde{A}+\mathrm{i} \lambda^{2} c_{32}|\tilde{A}|^{4} \tilde{A}+\mathcal{O}\left(|\tilde{A}|^{7}\right)+\tilde{\mathbf{E}}(t, a, \eta) .
$$

Here $\tilde{\mathbf{E}}=\mathbf{E} \circ(I+h)$, with $\mathbf{E}$ given by (4.49).

In (7.2),

$$
\gamma=\frac{3}{4} \frac{\lambda^{2}}{\Omega} \Gamma, \quad \Gamma \equiv \frac{\pi}{3 \Omega}\left(\mathbf{P}_{c} \varphi^{3}, \delta(B-3 \Omega) \mathbf{P}_{c} \varphi^{3}\right)>0 .
$$

The constants $c_{21}$ and $c_{32}$ are real numbers which are computable by the algorithm presented in Section 5.

The above definitions of $A, \tilde{A}$, and $h$, together with the estimates proved below, can be used to verify in a straightforward manner the assertions of Theorem 1.1 concerning $R(t) \equiv|A(t)|$, and $\theta(t) \equiv$ $\arg A(t)$.

To proceed with a study of the $t \rightarrow \infty$ behavior, recall that: 


$$
\begin{aligned}
\left(\partial_{t}^{2}+B^{2}\right) \eta_{1} & =0 \quad \eta_{1}(x, 0)=\mathbf{P}_{c} u_{0}, \partial_{t} \eta_{1}(x, 0)=\mathbf{P}_{c} u_{1} \\
\left(\partial_{t}^{2}+B^{2}\right) \eta_{2} & =\lambda a^{3} \mathbf{P}_{c} \varphi^{3}, \quad \eta_{2}(x, 0)=0, \partial_{t} \eta_{2}(x, 0)=0 \\
\left(\partial_{t}^{2}+B^{2}\right) \eta_{3} & =\lambda \mathbf{P}_{c}\left(3 a^{2} \varphi^{2} \eta+3 a \varphi \eta^{2}+\eta^{3}\right) \\
\eta_{3}(x, 0) & =\partial_{t} \eta_{3}(x, 0)=0 .
\end{aligned}
$$

To motivate the strategy, we first argue heuristically. Since $\gamma>0$, if $\tilde{\mathbf{E}}$ is negligible, then $|\tilde{A}| \sim\langle t\rangle^{-1 / 4}$ as $t \rightarrow \infty$, and therefore by (1.25), $a \sim\langle t\rangle^{-194}$, as $t \rightarrow \infty$. It then follows from (7.5) that, in appropriate norms, that $\eta_{2} \sim\langle t\rangle^{-3 / 4}$ and $\eta_{3} \sim\langle t\rangle^{-1+\delta}$ for some $\delta>0$.

To make all this precise requires á priori estimates on the above system of equations. We now recall Lemma 6.1 concerning equations of the form (7.2). This result gives conditions ensuring that $\tilde{A}$ behaves like the solution of the equation obtained by setting $\tilde{\mathbf{E}}$ to zero.

Our next task is to obtain an upper bound on $\tilde{\mathbf{E}}(t)$ in (4.49) of the form (6.7). Since the equation for $\tilde{A}(t)$ is coupled to that of $\eta$, the factor $Q_{0}$ in (6.7) will depend on $\eta$ and $\tilde{A}$. The next proposition, together with Proposition 4.7, provides estimates for the individual terms in $\tilde{\mathbf{E}}$.

It is convenient to introduce the notation:

$$
\begin{gathered}
{[A]_{\alpha}(T)=\sup _{0 \leq t \leq T}\langle t\rangle^{\alpha}|A(t)|} \\
{[\eta]_{p, \alpha}(T)=\sup _{0 \leq t \leq T}\langle t\rangle^{\alpha}|| \eta(t) \|_{p} .}
\end{gathered}
$$

To avoid cumbersome notation, where it should cause no confusion, we shall abbreviate expressions like $[\cdots](T)$ by $[\cdots]$, until it is necessary to make the dependence on $T$ explicit. In the estimates below, we shall often use the notation $C_{\varphi}$ to denote a constant depending on some $W^{k, p}$ norm of the bound state, $\varphi$. Under our hypotheses, $\varphi$ is a sufficiently smooth and exponentially decaying function for which these norms are finite; see [1].

Because of Proposition 5.1, we need only estimate the terms of $\mathbf{E}$, given in (4.49). The following proposition is the main step toward the estimate on $\mathbf{E}$.

Proposition 7.1. Estimates on the terms in $\mathbf{E}(t)$ : For $0 \leq s \leq t$, the terms of $\mathbf{E}(s)$, as defined in (4.49), are estimated as follows: 

(i) $\left|\lambda a \int \varphi \eta^{2}\right| \leq|\lambda| C_{\varphi}[A]_{1 / 4}[\eta]_{8,3 / 4}^{2}\langle t\rangle^{-7 / 4}$,
(ii) $\left|\lambda \int \varphi \eta^{3}\right| \leq \lambda C_{\varphi}[\eta]_{8,3 / 4}^{3}\langle t\rangle^{-9 / 4}$,
(iii) $\left|F_{2}\left(a, \eta_{1}\right)\right| \leq|\lambda|[A]_{1 / 4}^{2}\left\|\mathbf{u}_{0}\right\|_{\mathbf{x}}\langle t\rangle^{-13 / 8}$,
(iv) $\left|F_{2}\left(a, \eta_{3}\right)\right| \leq C_{\varphi}|\lambda|[A]_{1 / 4}^{2} C\left([A]_{1 / 4},[\eta]_{8,3 / 4},\left[B \eta_{3}\right]_{4,1 / 4+\sigma_{0}},\left\|\mathbf{u}_{0}\right\|_{\mathbf{x}}\right)$ $\times\langle t\rangle^{-5 / 4-\sigma_{0}}$,
(v) $\left|F_{2}\left(a, \eta_{*}^{n r 1}\right)\right| \leq C_{\varphi}|\lambda|^{2}\left|A_{0}\right|^{2}[A]_{1 / 4}^{2}\langle t\rangle^{-2}$,
(vi) $\left|F_{2}\left(a, \eta_{*}^{n r 2}\right)\right| \leq C_{\varphi}|\lambda|^{2}[A]_{1 / 4}^{2}\left[|A|^{2}\left|A^{\prime}\right|\right]_{5 / 4}\langle t\rangle^{-7 / 4}$
(vii) $\left|E_{2 j}^{n r}\right| \leq|\lambda| C_{\varphi}\left(\left[|A|^{4}\left|A^{\prime}\right|\right]_{7 / 4}+[A]_{1 / 4}^{2}+\left[|A|^{2}\left|\left(A^{3}\right)^{\prime \prime}\right|\right]_{7 / 4}\right)\langle t\rangle^{-13 / 8}$

In (iv), $C\left(r_{1}, r_{2}, r_{3}, r_{4}\right)$ is bounded for $\sum_{j}\left|r_{j}\right|$ bounded and tends to zero as $\sum_{j}\left|r_{j}\right|$ tends to zero; see Proposition 7.4.

We now embark on the proof of this proposition. Parts (i) and (ii) follow by Hölder's inequality. To prove part (iii), apply Hölders's inequality and the linear propagator estimates of Theorem 2.3. We now focus on (iv)-(vi).

\section{Estimation of $F_{2}\left(a, \eta_{3}\right)$}

Recall (see 4.18) that

$$
F_{2}\left(a, \eta_{3}\right)=3 \lambda a^{2} \int \varphi^{3} \eta_{3}
$$

We start by estimating $\left\|\eta_{3}\right\|_{8}$. We first express $\eta_{3}$, defined in (7.6), as

$$
\eta_{3}(t)=\lambda \sum_{j=1}^{3} \int_{I_{j}} E_{1}(t-s) \mathbf{P}_{c}\left(3 a^{2} \varphi^{2} \eta+3 a \varphi \eta^{2}+\eta^{3}\right) d s
$$

where

$$
I_{1}=[0, t / 2], I_{2}=[t / 2, t-1], \text { and } I_{3}=[t-1, t] .
$$

We estimate each integral separately. 


$$
\left\|\eta_{3}(t)\right\|_{8} \leq|\lambda| \sum_{j=1}^{3} \int_{I_{j}}\left\|E_{1}(t-s) \mathbf{P}_{c}\left(3 a^{2} \varphi^{2} \eta+3 a \varphi \eta^{2}+\eta^{3}\right)\right\|_{8} d s
$$

The integrands are estimated as follows using the linear estimates of Corollary 2.1:

$$
\begin{aligned}
& \int_{I_{1}}|| E_{1}(t-s) \mathbf{P}_{c}\{\cdots\}\left\|_{8} d s \leq C \int_{0}^{t / 2}|t-s|^{-9 / 8}\right\|\{\cdots\} \|_{1,8 / 7} d s \\
& \int_{I_{2}}|| E_{1}(t-s) \mathbf{P}_{c}\{\cdots\}\left\|_{8} d s \leq C \int_{t / 2}^{t-1}|t-s|^{-9 / 8}\right\|\{\cdots\} \|_{1,8 / 7} d s \\
& \left.\int_{I_{3}}|| E_{1}(t-s) \mathbf{P}_{c}\{\cdots\}\left\|_{8} d s \leq C \int_{t-1}^{t}|t-s|^{-3 / 8}\right\|\{\cdots\} \|_{1,8 / 7} d s\right)
\end{aligned}
$$

where

$$
\|\{\cdots\}\|_{1,8 / 7} \leq C\left(|A|^{2}\left\|\varphi^{2} \eta\right\|_{1,8 / 7}+|A|\left\|\varphi \eta^{2}\right\|_{1,8 / 7}+\left\|\eta^{3}\right\|_{1,8 / 7}\right)
$$

\section{Proposition 7.2.}

$$
\begin{aligned}
& \text { (i) }|A|^{2}\left\|\varphi^{2} \eta\right\|_{1,8 / 7} \leq C_{\varphi}|A|^{2}\left(\|\eta\|_{8}+\|B \eta\|_{4}\right), \\
& \text { (ii) }|A|\left\|\varphi \eta^{2}\right\|_{1,8 / 7} \leq C_{\varphi}|A|\left(\|\eta\|_{8}^{2}+\|B \eta\|_{4}\|\eta\|_{8}\right), \\
& \text { (iii) }\left\|\eta^{3}\right\|_{1,8 / 7} \leq C\left(\|\eta\|_{2,1,} \varphi\right)\|\eta\|_{8}^{5 / 3}
\end{aligned}
$$

Proof. We prove part (i). The estimates (ii) and (iii) follow similarly.

$$
\begin{aligned}
\left\|\varphi^{2} \eta\right\|_{1,8 / 7} & \leq C\left(\left\|\varphi^{2} \eta\right\|_{8 / 7}+\|\varphi \partial \varphi \eta\|_{8 / 7}+\left\|\varphi^{2} \partial \eta\right\|_{8 / 7}\right) \\
& \leq C\left(\left\|\varphi^{2}\right\|_{4 / 3}\|\eta\|_{8}+\|\varphi \partial \varphi\|_{4 / 3}\|\eta\|_{8}+\left\|\varphi^{2}\right\|_{8 / 5}\|\partial \eta\|_{4}\right)
\end{aligned}
$$

by Hölder's inequality. To express the right hand side of (7.23) in terms of $\|B \eta\|_{4}$, and thereby completing the proof of part (i), it suffices to show that: 


$$
\|\partial \eta\|_{4} \leq C\|B \eta\|_{4} .
$$

This follows if we show that the operator $\partial_{i} B^{-1}$ is bounded on $L^{p}$ (with $p=4$ ).

To prove the $L^{p}$ boundedness of $\partial_{i} B^{-1}$, we can apply the results on the wave operator, $W_{*}$ in $\S 2.2$. Indeed, for any $g \in L^{p}$, we have using the boundedness of the wave operators on $W^{1, p}$, for $p \geq 1$, that

$$
\begin{aligned}
\left\|\partial_{i} B^{-1} g\right\|_{p} & =\left\|\partial_{i} W_{+} B_{0}^{-1} W_{+}^{*} g\right\|_{p} \\
& \leq C\left\|W_{+} B_{0}^{-1} W_{+}^{*} g\right\|_{W^{1, p}} \\
& \leq C\left\|B_{0}^{-1} W_{+}^{*} g\right\|_{W^{1, p}} \\
& \leq C\left\|W_{+}^{*} g\right\|_{p} \leq C\|g\|_{p} .
\end{aligned}
$$

Remark. We offer here an alternative proof which does not make use of the wave operators, and therefore applies under weaker hypotheses on $V$. We begin with the square root formula see [49] and (2.33):

For any $\psi \in D\left(B^{2}\right)$ :

$$
B^{-1}=\pi^{-1} \int_{0}^{\infty} w^{-1 / 2}\left(B^{2}+w\right)^{-1} d w
$$

By (7.25) and the second resolvent formula we have:

$$
\partial_{i} B^{-1}=\partial_{i} B_{0}^{-1}+\pi^{-1} \int_{0}^{\infty} w^{-1 / 2} \partial_{i}\left(B_{0}^{2}+w\right)^{-1} V\left(B^{2}+w\right)^{-1} d w .
$$

The boundedness in $L^{p}(p \geq 1)$ of the operator $\partial_{i} B_{0}^{-1}$ holds because $\xi_{i}\left(|\xi|^{2}+m^{2}\right)^{-1 / 2}$ is a multiplier on $L^{p}$; see [64]. Similarly, $\partial_{i}\left(B_{0}^{2}+w\right)^{-1}$ is bounded on $L^{p}$ for any $w>0$ and estimation of the second term in (7.26) is reduced to estimation of the norm of the operator $V\left(B^{2}+w\right)^{-1}$. Note that:

$$
\left(B^{2}+w\right)^{-1}=\int_{0}^{\infty} \mathrm{e}^{-t\left(B^{2}+w\right)} d t
$$

Since inf $\sigma\left(B^{2}\right)=\Omega^{2}>0$, 


$$
\left\|\mathrm{e}^{-t\left(B^{2}-\Omega^{2} / 2\right)}\right\|_{\mathscr{B}\left(L^{p}\right)} \leq C
$$

and therefore

$$
\left\|\left(B^{2}+w\right)^{-1}\right\|_{\mathscr{B}\left(L^{p}\right)} \leq C \int_{0}^{\infty} \mathrm{e}^{-t\left(\Omega^{2} / 2+w\right)} \leq C^{\prime}(1+w)^{-1} .
$$

Boundedness in $L^{p}$ of $\partial_{i} B^{-1}$ now follows. Namely,

$$
\begin{aligned}
\left\|\partial_{i} B^{-1}\right\|_{\mathscr{B}\left(L^{p}\right)} \leq & \left\|\partial_{i} B_{0}^{-1}\right\|_{\mathscr{B}\left(L^{p}\right)} \\
& +C^{\prime}\left\|\partial_{i}\left(B_{0}^{2}+w\right)^{-1}\right\|_{\mathscr{B}\left(L^{p}\right)}\|V\|_{\infty} \\
& \times \int_{0}^{\infty} w^{-1 / 2}(1+w)^{-1} d w<\infty .
\end{aligned}
$$

From (7.22) we see that $\|B \eta\|_{4}$ must be estimated. Recall that

$$
B \eta=B \eta_{1}+B \eta_{2}+B \eta_{3} .
$$

By (7.4)

$$
B \eta_{1}=E_{0}(t) B \mathbf{P}_{c} u_{0}+E_{1}(t) B \mathbf{P}_{c} u_{1}
$$

and so

$$
\left\|B \eta_{1}(t)\right\|_{4} \leq C\langle t\rangle^{-1 / 2}\left\|\mathbf{u}_{0}\right\|_{\mathbf{x}}
$$

see (3.7) for definition of $\left\|\mathbf{u}_{0}\right\|_{\mathbf{x}}$.

By (7.5)

$$
B \eta_{2}=\lambda \int_{0}^{t} E_{1}(t-s) a^{3}(s) B \mathbf{P}_{c} \varphi^{3} d s
$$

which can by Theorem 2.3 be estimated as

$$
\begin{aligned}
\left\|B \eta_{2}(t)\right\|_{4} & \leq C|\lambda| \int_{0}^{t}|t-s|^{-3 / 4}|A(s)|^{3}|| \mathbf{P}_{c} \varphi^{3} \|_{4 / 3} d s \\
& \leq C_{\varphi}|\lambda|[A]_{1 / 4}^{3}\langle t\rangle^{-1 / 2}
\end{aligned}
$$


The estimation of $\left\|B \eta_{3}(t)\right\|_{4}$ is more involved. By (7.6),

$$
B \eta_{3}=\lambda \int_{0}^{t} \sin B(t-s) \mathbf{P}_{c}\left[3 a^{2} \varphi^{2} \eta+3 a \varphi \eta^{2}+\eta^{3}\right] d s .
$$

By Theorem 2.3,

$$
\begin{aligned}
\left\|B \eta_{3}(t)\right\|_{4} \leq C|\lambda| \int_{0}^{t}|t-s|^{-1 / 2}\left[\left[|A|^{2}\left\|\varphi^{2} \eta\right\|_{1,4 / 3}\right.\right. \\
\left.+|A|\left\|\varphi \eta^{2}\right\|_{1,4 / 3}+\left\|\eta^{3}\right\|_{1,4 / 3}\right] d s .
\end{aligned}
$$

The following proposition provides estimates for the integrand. It is proved in the same manner as Proposition 7.2.

\section{Proposition 7.3.}

$$
\begin{aligned}
& |A|^{2}\left\|\varphi^{2} \eta\right\|_{1,4 / 3} \leq C_{\varphi}|A|^{2}\left(\|\eta\|_{8}+\left\|B \eta_{1}\right\|_{4}+\left\|B \eta_{2}\right\|_{4}+\left\|B \eta_{3}\right\|_{4}\right) \\
& |A|\left\|\varphi \eta^{2}\right\|_{1,4 / 3} \leq C_{\varphi}|A|\left(\|\eta\|_{8}^{2}+\|\eta\|_{8}\|B \eta\|_{4}\right) \\
& \left\|\eta^{3}\right\|_{1,4 / 3} \leq 3\|\eta\|_{8}^{2}\|\partial \eta\|_{2}
\end{aligned}
$$

Anticipating the behavior

$$
|A(t)| \sim t^{-1 / 4},\|\eta(t)\|_{8} \sim t^{-3 / 4},
$$

and using (7.33), we find that $B \eta_{3}$ is driven by terms which are formally of order $\langle t\rangle^{-1}$. Estimation in $L^{4}$ will lead to convolution of $\langle t\rangle^{-1}$ with $\langle t\rangle^{-1 / 2}$ giving a rate of $\langle t\rangle^{-1 / 2+\delta_{0}}$, for any $\delta_{0}>0$.

For $0 \leq t \leq T$, with $T$ fixed and arbitrary, we have:

$$
\begin{aligned}
\left|A^{2}\right|\left\|\varphi^{2} \eta\right\|_{4 / 3,1} \leq & C_{\varphi}[A]_{1 / 4}^{2}\left([\eta]_{8,3 / 4}\langle t\rangle^{-5 / 4}+\left[B \eta_{1}\right]_{4,1 / 2}\langle t\rangle^{-1}\right. \\
& \left.+\left[B \eta_{2}\right]_{4,1 / 2}\langle t\rangle^{-1}+\left[B \eta_{3}\right]_{4,1 / 2-\delta_{0}}\langle t\rangle^{-1+\delta_{0}}\right)
\end{aligned}
$$

where $\delta_{0}$ is positive and arbitrary. Also,

$$
\begin{aligned}
|A|\left\|\varphi \eta^{2}\right\|_{1,4 / 3} & \leq C_{\varphi}[A]_{1 / 4}\left([\eta]_{8,3 / 4}^{2}\langle t\rangle^{-7 / 4}+[\eta]_{8,3 / 4}\left[B \eta_{1}\right]_{4,0}\langle t\rangle^{-1}\right) \\
\left\|\eta^{3}\right\|_{1,4 / 3} & \leq\|\eta\|_{1,2}[\eta]_{8,3 / 4}^{2}\langle t\rangle^{-3 / 2} .
\end{aligned}
$$


It follows that for $0 \leq t \leq T$

$$
\left\|B \eta_{3}\right\|_{4} \leq C_{\varphi}|\lambda| \int_{0}^{t}|t-s|^{-1 / 2} d s\langle t\rangle^{-1+\delta_{0}} G(A, \eta, T)
$$

where

$$
\begin{aligned}
G(A, \eta, T) \equiv & {[A]_{1 / 4}^{2}\left([\eta]_{8,3 / 4}+\left[B \eta_{1}\right]_{4,1 / 2}+\left[B \eta_{2}\right]_{4,1 / 2}+\left[B \eta_{3}\right]_{4,1 / 2-\delta_{0}}\right) } \\
& +[A]_{1 / 4}\left([\eta]_{8,3 / 4}^{2}+[\eta]_{8,3 / 4}\left[B \eta_{1}\right]_{4,0}\right)+\|\eta\|_{1,2}[\eta]_{8,3 / 4}^{2} .
\end{aligned}
$$

Therefore, for $0 \leq t \leq T$ :

$$
\left\|B \eta_{3}(t)\right\|_{4} \leq C_{\varphi}|\lambda| G(A, \eta, T)\langle t\rangle^{-1 / 2+\delta_{0}}
$$

for any $\delta_{0}>0$. We now use the estimate (7.40) in (7.22) in order to obtain a bound on $\left\|\eta_{3}(t)\right\|_{8}$. First, a consequence of (7.22), (7.33), (7.35) and (7.40) is that for $0 \leq t \leq T$ :

$$
\begin{gathered}
|A|^{2}\left\|\varphi^{2} \eta\right\|_{1,8 / 7} \leq C_{\varphi}[A]_{1 / 4}^{2}\left([\eta]_{8,3 / 4}+\left\|\mathbf{u}_{0}\right\|_{\mathbf{x}}+[A]_{1 / 4}^{3}+\left[B \eta_{3}\right]_{4,1 / 2-\delta_{0}}\right) \\
\langle t\rangle^{-1+\delta_{0}}
\end{gathered}
$$

$|A|\left\|\varphi \eta^{2}\right\|_{1,8 / 7} \leq C_{\varphi}[A]_{1 / 4}\left([\eta]_{8,3 / 4}^{2}+\left\{\left\|\mathbf{u}_{0}\right\|_{\mathbf{x}}+\left[B \eta_{3}\right]_{4,1 / 2-\delta_{0}}\right.\right.$

$$
\begin{aligned}
\left.\left.+[A]_{1 / 4}^{3}\right\}[\eta]_{8,3 / 4}\right)\langle t\rangle^{-1+\delta_{0}}, \\
\left\|\eta^{3}\right\|_{1,8 / 7} \leq C_{\varphi}\left(\|\eta\|_{1,2}\right)[\eta]_{8,3 / 4}^{5 / 3}\langle t\rangle^{-5 / 4} .
\end{aligned}
$$

Substitution of (7.41) into (7.17-7.20) leads to an estimate for $\left\|\eta_{3}(t)\right\|_{8}$. For $0 \leq t \leq T$ :

$$
\begin{aligned}
\left\|\eta_{3}(t)\right\|_{8} \leq & |\lambda| C_{\varphi}\langle t\rangle^{-1+\delta_{0}}\left([A]_{1 / 4}^{2}\left\{[\eta]_{8,3 / 4}+\left\|\mathbf{u}_{0}\right\|_{\mathbf{x}}+[A]_{1 / 4}^{3}+\left[B \eta_{3}\right]_{4,1 / 2-\delta_{0}}\right\}\right. \\
& +[A]_{1 / 4}\left\{[\eta]_{8,3 / 4}^{2}+\left[\left\|\mathbf{u}_{0}\right\|_{\mathbf{x}}+\left[B \eta_{3}\right]_{4,1 / 2-\delta_{0}}+[A]_{1 / 4}^{3}\right][\eta]_{8,3 / 4}\right\} \\
& \left.+C\left(\|\eta\|_{1,2}, \varphi\right)[\eta]_{8,3 / 4}^{5 / 3}\right),
\end{aligned}
$$

where $\delta_{0}$ is arbitrary. 
Finally we can now estimate $F_{2}\left(a, \eta_{3}\right)$ using (4.18) and (7.42). Choosing $\delta_{0}$ so that

$$
-1+\delta_{0}=-3 / 4-\sigma_{0}, \text { with } \sigma_{0}>0,
$$

we have

\section{Proposition 7.4.}

$$
\begin{aligned}
&\left|F_{2}\left(a, \eta_{3}\right)\right| \leq C_{\varphi}|\lambda|[A]_{1 / 4}^{2}\left([A]_{1 / 4}^{2}\left\{[\eta]_{8,3 / 4}+\left\|\mathbf{u}_{0}\right\|_{\mathbf{x}}+[A]_{1 / 4}^{3}+\left[B \eta_{3}\right]_{4,1 / 4+\sigma_{0}}\right\}\right. \\
&+ {[A]_{1 / 4}\left\{[\eta]_{8,3 / 4}^{2}+\left[\left\|\mathbf{u}_{0}\right\|_{\mathbf{x}}+[A]_{1 / 4}^{3}+\left[B \eta_{3}\right]_{4,1 / 4+\sigma_{0}}\right][\eta]_{8,3 / 4}\right\} } \\
&+\left.C\left(\|\eta\|_{1,2}, \varphi\right)[\eta]_{8,3 / 4}^{5 / 3}\right)\langle t\rangle^{-5 / 4-\sigma_{0}} . \\
& \text { Estimation of } F_{2}\left(a, \eta_{*}^{\text {nr1 }}\right) \text { and } F_{2}\left(a, \eta_{*}^{n r 2}\right)
\end{aligned}
$$

By (4.23)

$$
\begin{aligned}
& \eta_{*}^{n r 1}=-\frac{\lambda}{2} A_{0}^{3} B^{-1}(B-3 \Omega+\mathrm{i} 0)^{-1} \mathrm{e}^{\mathrm{i} B t} \mathbf{P}_{c} \varphi^{3}, \text { and } \\
& \eta_{*}^{n r 2}=-\frac{3 \lambda}{2} B^{-1}(B-3 \Omega+\mathrm{i} 0)^{-1} \int_{0}^{t} \mathrm{e}^{\mathrm{i} B(t-s)} \mathrm{e}^{\mathrm{i} 3 \Omega s} A^{2} A^{\prime} d s \mathbf{P}_{c} \varphi^{3}
\end{aligned}
$$

Estimation using Proposition 2.2 gives:

$$
\begin{aligned}
\left|F_{2}\left(a, \eta_{*}^{n r 1}\right)\right|= & 3|\lambda||a|^{2}\left|\int \varphi^{3} \eta_{*}^{n r 1}\right| \\
= & C|\lambda|^{2}\left|A_{0}\right|^{3}|A|^{2}\left|\int \varphi^{3}[B(B-3 \Omega+\mathrm{i} 0)]^{-1} \mathrm{e}^{\mathrm{i} B t} \mathbf{P}_{c} \varphi^{3}\right| \\
\leq & C|\lambda|^{2}\left|A_{0}\right|^{3}|A|^{2}||\langle x\rangle^{\sigma} \varphi^{3} \|_{2}||\langle x\rangle^{-\sigma} \\
& \times(B-3 \Omega+\mathrm{i} 0)^{-1} \mathrm{e}^{\mathrm{i} B t} \mathbf{P}_{c} B^{-1} \varphi^{3} \|_{2} \\
\leq & C_{\varphi}|\lambda|^{2}\left|A_{0}\right|^{2}[A]_{1 / 4}^{2}\langle t\rangle^{-\frac{5}{4}+\delta} .
\end{aligned}
$$


Here, we have used that $1 / 2+6 / 5=5 / 4+\delta$, for some $\delta>0$. The constant $C_{\varphi}$ depends on $\left\|\langle x\rangle^{\sigma} B^{-1}\langle x\rangle^{-\sigma}\right\|_{\mathscr{B}\left(L^{2}\right)}$. It is easy to check that this norm is bounded if we replace $B^{-1}$ by $B^{-2}$. The estimate of interest is reduced to this case using the Kato square root formula (7.25). Similarly, we have

$$
\begin{aligned}
\left|F_{2}\left(a, \eta_{*}^{n r 2}\right)\right| & =3|\lambda||a|^{2}\left|\int \varphi^{3} \eta_{*}^{n r 2}\right| \\
& =C_{\varphi}|\lambda|^{2}|A|^{2} \mid \int \varphi^{3}[B(B-3 \Omega+\mathrm{i} \varepsilon)]^{-1} \\
& \int_{0}^{t} \mathrm{e}^{\mathrm{i}(t-s)(B-3 \Omega)} A^{2} A^{\prime} d s \mathbf{P}_{c} \varphi^{3} \mid \\
& \leq C_{\varphi}|\lambda|^{2}|A|^{2} \int_{0}^{t}\langle t-s\rangle^{-\frac{3}{2}+\mu}|A|^{2}\left|A^{\prime}\right| d s \\
& \leq C_{\varphi}|\lambda|^{2}[A]_{1 / 4}^{2}\left[|A|^{2}\left|A^{\prime}\right|\right]_{5 / 4}\langle t\rangle^{-\frac{7}{4}},
\end{aligned}
$$

where we have taken $\mu$ sufficiently small so that the last integral $t$ integral is convolution with an $L^{1}$ function, which then preserves the decay rate, $\langle t\rangle^{-\frac{7}{4}}$, which exceeds $\langle t\rangle^{-\frac{5}{4}}$

\section{Estimation of $E_{2 j}^{n r}$ :}

To estimate (4.47) we use Propositions 2.1 and 2.2. Due to the singularity in the resolvent at frequency $3 \Omega$, the case $j=7$, is most difficult and we focus on it. To treat this singularity, we use Proposition 2.2, for $n=3$ :

$$
\left\|\langle x\rangle^{-\sigma}\left(B-\zeta_{7}\right)^{-2} \mathrm{e}^{\mathrm{i} B(t-s)}\langle x\rangle^{-\sigma} \psi\right\|_{2} \leq C\langle t-s\rangle^{-\frac{6}{5}}\|\psi\|_{1,2} .
$$

Use of this estimate in (4.47) yields

$$
\begin{gathered}
\left|E_{2 j}^{n r}\right| \leq C_{\varphi}|\lambda|^{2}\left(\left[|A|^{4}\left|A^{\prime}\right|\right]_{7 / 4}\langle t\rangle^{-\frac{7}{4}}+[A]_{1 / 4}^{2}\langle t\rangle^{-\frac{13}{8}}+\left[|A|^{4}\left|A^{\prime \prime}\right|\right.\right. \\
\left.\left.+\left|A^{3}\right|\left|A^{\prime}\right|^{2}\right]_{9 / 4}\langle t\rangle^{-\frac{13}{8}}\right)
\end{gathered}
$$

from which estimate (vii) of Proposition 7.1 follows. This finally completes the proof of Proposition 7.1. 
Proposition 7.1 can now be used to obtain the desired estimate for $\mathbf{E}(t)$ and therefore $\tilde{A}(t)$, for $|A|$ sufficiently small. Note that the right hand side of the estimates depend on $\left|A^{\prime}\right|$ and $\left|A^{\prime \prime}\right|$. These can each be estimated directly from the equation for $A,(4.48)$, and its derivative. The result is that $\left|A^{\prime}\right|$ and $\left|A^{\prime \prime}\right|$ may be estimated by $[A]_{1 / 4}^{3}\langle t\rangle^{-\frac{3}{4}}$, the only effect being a change in the multiplicative constants which is independent of $A$ and $\eta$. This observation together with Proposition 7.1 yields:

Proposition 7.5. There is positive number $\delta$ such that

$$
|\mathbf{E}(t)| \leq Q_{0}(A, \eta)\langle t\rangle^{-\frac{5}{4}-\delta}
$$

where

$$
Q_{0}(A, \eta)=[\eta]_{8,3 / 4}^{3}+[A]_{1 / 4}\left(1+C\left([A]_{1 / 4},[\eta]_{8,3 / 4},\left[B \eta_{3}\right]_{4,1 / 4+\sigma_{0}},\left\|\mathbf{u}_{0}\right\| \|_{\mathbf{x}}\right)\right)
$$

It now remains to estimate $\|\eta\|_{8}$.

$$
\begin{aligned}
\|\eta\|_{8} \leq & \left\|\eta_{1}\right\|_{8}+\left\|\eta_{2}\right\|_{8}+\left\|\eta_{3}\right\|_{8} \\
\leq & \left\|E_{0}(t) \mathbf{P}_{c} u_{0}\right\|_{8}+\left\|E_{1}(t) \mathbf{P}_{c} u_{1}\right\|_{8} \\
& +|\lambda| \int_{0}^{t}\left\|\mathbf{E}_{1}(t-s) a^{3}(s) \mathbf{P}_{c} \varphi^{3}\right\|_{8} d s+\left\|\eta_{3}\right\|_{8} .
\end{aligned}
$$

Using Theorem 2.3 and the bound (7.42) we get

\section{Proposition 7.6.}

$$
\begin{aligned}
\|\eta\|_{8} \leq & C_{\varphi}\left(\left\|\mathbf{u}_{0}\right\|_{\mathbf{x}}+[A]_{1 / 4}^{3}\right. \\
& +[A]_{1 / 4}^{2}\left\{[\eta]_{8,3 / 4}+\left\|\mathbf{u}_{0}\right\|_{\mathbf{x}}+[A]_{1 / 4}^{3}+\left[B \eta_{3}\right]_{4,1 / 4+\sigma_{0}}\right\} \\
& +[A]_{1 / 4}\left\{[\eta]_{8,3 / 4}^{2}+\left[\left\|\mathbf{u}_{0}\right\|_{\mathbf{x}}+[A]_{1 / 4}^{3}+\left[B \eta_{3}\right]_{4,1 / 4+\sigma_{0}}\right][\eta]_{8,3 / 4}\right\} \\
& \left.+C\left(\|\eta\|_{1,2,} \varphi\right)[\eta]_{8,3 / 4}^{5 / 3}\right)\langle t\rangle^{-3 / 4}
\end{aligned}
$$

The following proposition summarizes our labors. 
Proposition 7.7. For any $T>0$ :

$$
\begin{aligned}
{[\eta]_{8,3 / 4}(T) \leq } & C_{\varphi}\left(\left\|\mathbf{u}_{0}\right\|_{\mathbf{x}}+[A]_{1 / 4}^{3}(T)+\left[B \eta_{3}\right]_{4,1 / 4+\sigma}^{2}(T)\right. \\
& \left.+C_{\varphi}\left(\|\eta\|_{1,2}\right)[\eta]_{8,3 / 4}^{5 / 3}(T)\right) \\
{[A]_{1 / 4}^{4}(T) \leq } & \left(\left|A_{0}\right|^{4}+Q_{0}(A, \eta)^{\frac{8}{5}}\right) \\
{\left[B \eta_{3}\right]_{4,1 / 4+\sigma_{0}}(T) \leq } & \left([A]_{1 / 4}^{2}(T)+[\eta]_{8,3 / 4}^{2}(T)+\left[B \eta_{2}\right]_{4,1 / 2}^{2}(T)\right. \\
& \left.+\left[B \eta_{3}\right]_{4,1 / 4+\sigma_{0}}^{2}(T)\right), \\
\sup _{0 \leq t \leq T}\|\eta(t)\|_{1,2} \leq & C \mathscr{E}\left(u_{0}, u_{1}\right) \leq C\left\|u_{0}, u_{1}\right\|_{1,2} .
\end{aligned}
$$

The first three estimates are proved above while the last follows from conservation of energy (see section 1) and the decompostion of the solution.

Now define

$$
M(T) \equiv[\eta]_{8,3 / 4}(T)+[A]_{1 / 4}(T)+\left[B \eta_{3}\right]_{4,1 / 4+\sigma_{0}}(T) .
$$

Then, combining the estimates of the previous proposition we have, for some $\alpha>0$ :

$$
M(T)\left(1-M(T)^{\alpha}\right) \leq C_{\varphi}\left\|\mathbf{u}_{0}\right\|_{\mathbf{x}} .
$$

If $M(0)$ and $\left\|\mathbf{u}_{0}\right\|_{\mathbf{x}}$ are sufficiently small, we have by the continuity of $M(T)$, that there is a constant $M_{*}$, which is independent of $T$, such that for all $\mathrm{T}$

$$
M(T) \leq M_{*} .
$$

This completes the proof of Theorem 1.1.

\section{Summary and discussion}

We have considered a class of nonlinear Klein-Gordon equations, (1.1), which are perturbations of a linear dispersive equation which has a time periodic and spatially localized (bound state) solution. The unperturbed and perturbed dynamical systems are Hamiltonian. We have shown that if a nonlinear resonance condition (1.8) holds, then solutions with sufficiently small initial data tend to zero as $t \rightarrow \pm \infty$. This resonance condition is a nonlinear variant of the Fermi golden rule (1.22). A consequence of our result is that time-periodic and spatially localized solutions do not persist under small Hamiltonian 
perturbations. Time-decay of small amplitude solutions is also a property of the translation invariant $(V \equiv 0)$ nonlinear KleinGordon equation. However, the presence of a bound state of the unperturbed problem, causes a nonlinear resonance leading to the anomalously slow radiative decay of solutions.

We now conclude with some further remarks on the results of this paper, mention directions currently under investigation and some open problems.

\section{Anomalously slow time-decay rates:}

It is natural to compare the time-decay rate of solutions described by Theorem 1.1 with those of related problems.

(1a) Translation invariant linear Klein-Gordon equation $(V \equiv 0$ and $\lambda=0)$ :

We shall refer to free dispersive rates of decay as those associated with the constant coefficient equation:

$$
\partial_{t}^{2} u-\Delta u+m^{2} u=0 .
$$

Results on this are presented in section 2 . Roughly speaking, if the initial data has a sufficient number of derivatives in $L^{p}, 1<p \leq 2$, then the solution decays at a rate $\mathcal{O}\left(t^{-n\left(\frac{1}{2}-\frac{1}{p^{p}}\right)}\right)$ in $L^{p^{\prime}}$. Here, $p^{-1}+\left(p^{\prime}\right)^{-1}=1$.

(1b) Translation invariant nonlinear Klein Gordon equation $(V \equiv 0$ and $\lambda \neq 0$ ):

For small initial conditions it has been shown that solutions decay at free dispersive rates; see [65] and references cited therein.

(1c) Linear Klein-Gordon equation with a potential having a bound state, as hypothesized ( $V \neq 0$ and $\lambda=0)$ : By the spectral theorem, a typical solution will decompose into a linear superposition of (i) a bound state part, of the form: $R_{0} \cos \left(\Omega t+\rho_{0}\right) \varphi(x)$, and (ii) a part which disperses to zero at free dispersive rates.

(1d) Nonlinear Klein-Gordon equation with a potential having a bound state, as hypothesized $(V \neq 0$ and $\lambda \neq 0)$ : Whereas the decaying part of the solution in the above cases tends to zero at a free dispersive rate, Theorem 1.1 implies that the decay rate is anomalously slow. In particular, the decay rate obtained in $L^{8}$ is $\mathcal{O}\left(t^{-\frac{1}{4}}\right)$, while the free dispersive rate in $L^{8}$ is $\mathcal{O}\left(t^{-\frac{9}{8}}\right)$. This slow rate of decay, due to the nonlinear resonant interactions, gives rise to a long-lived or metastable states.

\section{Dispersive Hamiltonian normal form}

In a finite dimensional Hamiltonian system of one degree of freedom, the general normal form is: 


$$
A^{\prime}=\mathrm{i}\left(c_{10}+c_{21}|A|^{2}+c_{32}|A|^{4}+\cdots+c_{n+1, n}|A|^{2 n}+\cdots\right) A,
$$

where $c_{n, n+1}$ are real numbers. In present context we have the dispersive Hamiltonian normal form

$$
A^{\prime}=\mathrm{i}\left(k_{10}+k_{21}|A|^{2}+k_{32}|A|^{4}+\cdots+k_{n+1, n}|A|^{2 n}+\cdots\right) A,
$$

where

$$
k_{n+1, n}=d_{n+1, n}+\mathrm{i} c_{n+1, n}
$$

are, in general, numbers with real and imaginary part. Contributions to the real parts of coefficients come from resonances with the continuous spectrum. If (1.8) fails there are two possibilities; either (a) $3 \Omega$ does not lie in the continuous spectrum of $B$ (it lies in the gap $(0, m)$ ), or (b) $3 \Omega$ lies in the continuous spectrum of $B$ but we are in the nongeneric situation where the projection of $\varphi^{3}$ onto the generalized (continuum) eigenmode of frequency $3 \Omega$ is zero. In either case, we expect that, typically, internal dissipation would arise in the normal form at higher order. More precisely, we conjecture that the leading order nonzero $d_{n_{*}+1, n_{*}}$, which would correspond to a resonance with the continuum of a higher harmonic: $q_{*} \Omega \in \sigma_{\text {cont }}(B), q_{*}>3$ is always negative. (If the sign of $d_{n_{*}+1, n_{*}}$ were positive, this would seem to be in violation of the conservation of energy and the implied Lyapunov stability of the zero solution.) The corresponding decay rate would then be slower, specifically $\mathcal{O}\left(|t|^{-\frac{1}{2 n_{*}}}\right)$.

From this perspective one may expect the existence of breather solutions of integrable non-linear flows like the sine-Gordon equation or the extremely long-lived breather -like states of the $\phi^{4}$-model as corresponding to the case where to all order the coefficients $d_{n+1, n}$ are zero.

Does the vanishing of all such coefficients have an interpretation in terms of the infinitely many time-invariants for the integrable flow?

\section{Multiple bound state problems:}

Of interest are problems where the underlying potential, $V(x)$, supports more than one bound state. What is the large time behavior of such systems? Our analysis and the above remarks suggest that a more general normal form could be developed, and the corresponding (in general slower) decay rates anticipated. Is it possible that a resonance among the multiple "discrete oscillators" can be arranged so that one gets a persistence of nondecaying solutions, or does radiation to the continuum always win out? 


\section{Relation to center manifold theory:}

Our program of decomposing the original conservative dynamical system into a finite dimensional dynamical system (1.20) which is weakly coupled to an infinite dimensional dynamical system is one commonly used in dissipative dynamical systems. There, it is often possible using the center manifold approach [14], [67], to construct an invariant center-stable manifold. The dynamics in a neighborhood of an equilibrium point are characterized by an exponentially fast contraction on to the invariant manifold. The contraction is exponential because the part of the linearized spectrum associated with the infinite dimensional part of the dynamics is contained in the left half plane. In the current context of conservative dynamical systems, the linearization about the equilibrium point (here $u \equiv 0$ ) lies on the imaginary axis; in particular, two complex conjugate eigenvalues $\pm \mathrm{i} \Omega$ and continuous spectrum from $\pm \mathrm{i} m$ to $\pm \mathrm{i} \infty$. The analogue of dissipation is the mechanism of dispersive radiation of energy, related to the continuous spectrum, and the associated algebraic time-decay.

There is recent work on the application of center manifold methods to certain special conservative dynamical systems of nonlinear Schrödinger type; see the center manifold analysis of [46] applied to problem studied by the authors in [60]. It would be of interest to understand whether geometric insight on the structure of the phase space for problems of the type considered in this paper can be obtained using the ideas of invariant manifold theory.

\section{Systems with disorder:}

In this paper, we have seen the effect of a single localized defect on the wave propagation dynamics in a nonlinear system. Of great interest would be an understanding of the effects of a spatially random distribution of defects modeled, for example, by random potential $V(x)$ on the localization of energy in nonlinear systems such as (1.1). Related questions are studied in [22], [25], [10].

Acknowledgements. The problem studied in this paper was raised by T.C. Spencer in lectures and informal discussions and by J. Fröhlich in the late 1980's. M.I.W. learned of this problem from T.C. Spencer and A.S. from I.M. Sigal and J. Fröhlich. The authors wish to thank them for their insights and continued interest. The authors also wish to thank B. Birnir for stimulating discussions, and R. Pyke, J.B. Rauch and an anonymous referee for their careful reading of and thoughtful comments on the manuscript. This research was carried out while MIW was on sabbatical leave in the Program in Applied and Computational Mathematics at Princeton University. MIW would like to thank Phil Holmes for his hospitality and for providing a stimulating research environment. This research was supported in 
part by NSF grant DMS-9401777 and an FAS-Rutgers grant award (AS) and by NSF grant DMS- 9500997 (MIW).

\section{References}

[1] Agmon, S.: Lectures on Exponential Decay of Solutions of Second-Order Elliptic Equations: Bounds on Eigenfunctions of N-Body Schrödinger Operators, Princeton University Press (1982)

[2] Aichelburg, P.C., Beig, R.: Radiation damping as an initial value problem, Ann. Phys. 98, 264-283 (1976)

[3] Arnol'd, V.I.: Geometric Methods in the Theory of Ordinary Differential Equations, Springer-Verlag, New York (1983)

[4] Bourgain, J.: Quasi-periodic solutions of Hamiltonian evolution equations, Lecture Notes in Pure and Appl. Math. 186, Dekker, New York 1997

[5] Brenner, P.: On the existence of global smooth solutions of certain semilinear hyperbolic equations, Math. Z. 167 99-135; On scattering and everywhere defined scattering operators for nonlinear Klein-Gordon equations, J. Diff. Eqns. 56, (1985) 310-344 (1979)

[6] Brezis, H.: Periodic solutions of nonlinear vibrating strings and duality principles, Bull. Amer. Math. Soc. (N.S.) 8, 409-426 (1983)

[7] Bonilla, L., Keller, J.B.: Irreversibility and nonrecurrence, J. Stat. Phys. 42, 1115-1125 (1986)

[8] Birnir, B., McKean, H.P., Weinstein, A.: The rigidity of sine-Gordon breathers, Comm. Pure Appl. Math. 47, 1043-1051 (1994)

[9] Birnir, B.: Qualitative analysis of radiating breathers, Comm. Pure Appl. Math. 47, 103-119 (1994)

[10] Bronski, J.C.: A numerical study of solitary wave propagation in a disordered medium, preprint

[11] Buslaev, V.S., Perel'man, G.S.: Scattering for the nonlinear Schrödinger equation: states close to a soliton, St. Petersburg Math. J. 4, 1111-1142 (1993)

[12] Buslaev, V.S., Perel'man, G.S.: On the stability of solitary waves for nonlinear Schrödinger equation, Amer. Math. Soc. Transl. Ser. 2 164, 75-98

[13] Campbell, D.K., Peyrard, M.: Solitary wave collisions revisited, Physica D 18, 47 (1986)

[14] Carr, J.: Applications of Centre Manifold Theory, Springer-Verlag, New York (1981)

[15] Coddington, E., Levinson, N.: Theory of Ordinary Differential Equations, McGraw-Hill, New York, 1955

[16] Coron, J.M.: Periode minimale pour une corde vibrante de longeur infinie, C.R. Acad. Sci. A294, 127-129 (1982)

[17] Craig, W., Wayne, C.E.: Newton's method and periodic solutions of nonlinear wave equations, Comm. Pure Appl. Math. 46, 1409-1498 (1993)

[18] Cycon, H.L., Froese, R.G., Kirsch, W., Simon, B.: Schrödinger Operators, Springer-Verlag, Berlin Heidelberg New York, 1987

[19] Denzler, J.: Nonpersistence of breather families for the perturbed Sine-Gordon equation, Commun. Math. Phys. 158, 397- 430 (1993)

[20] Debiévre, S., Hislop, P.D., Sigal, I.M.: Scattering theory for the wave equation on non-compact manifolds, Rev. Math. Phys. 4, 575-618 (1992) 
[21] Ford, G., Kac, M., Mazur, P.: Statistical properties of assemblies of coupled oscillators J. Math. Phys. 6, 504-515 (1965)

[22] Fröhlich, J., Spencer, T., Wayne, C.E.: Localization in disordered nonlinear dynamical systems, J. Stat. Phys. 42, 247-274 (1986)

[23] Ginibre, J., Velo, G.: Scattering theory in the energy space for a class of nonlinear wave equations, Comm. Math. Phys. 123, 535-573 (1989)

[24] Grillakis, M., Shatah, J., Strauss, W.: Stability theory of solitary waves in the presence of symmetry, I, J. Func. Anal. 74, 160-197 (1987)

[25] Gredeskul, S.A., Kivshar, Y.S., Sanchez, A., Vazquez, L.: Localization decay induced by strong nonlinearity in disordered systems, Phys. Rev. Lett. 64, 1693-1696 (1990)

[26] Guckenheimer, J., Holmes, P.: Nonlinear Oscillations, Dynamical Systems, and Bifurcations of Vector Fields, Springer-Verlag (1983)

[27] Gérard, C., Sigal, I.M.: Space-time picture of semiclassical resonances, Commun. Math. Phys. 145, (1992) 281-328

[28] Hunziker, W., Sigal, I.M.: Time dependent scatterinig theory for N-body quantum systems, ETH-Zürich preprint (1997)

[29] Hunziker, W., Sigal, I.M., Soffer, A.: Minimal escape velocities, ETH-Zürich preprint (1997)

[30] Jaksić, V., Pillet, C.-A.: On a model for quantum friction. I. Fermi's golden rule and dynamics at zero temperature, Ann. Inst. H. Poincaré Phys. Théor. 62, $47-$ 68 (1995)

[31] Jensen, A., Kato, T.: Spectral properties of Schrödinger operators and timedecay of wave functions, Duke Math. J. 46 583-611 (1979)

[32] Jona-Lasinio, G., Presilla, C., Sjöstrand, J.: On Schrödinger equations with concentrated nonlinearities, Ann. Phys. 240, (1995)

[33] Journé, J.-L., Soffer, A., Sogge, C.D.: Decay estimates for Schrödinger operators, Comm. Pure Appl. Math. 44, 573-604 (1991)

[34] Kichenassamy, S.: Breather solutions of nonlinear wave equations, Commun. Pure Appl. Math. 44, 789 (1991)

[35] Kivshar, Yu.S., Malomed, B.A.: Rev. Mod. Phys. 61, 763 (1989)

[36] Klainerman, S.: Remark on the asymptotic bahavior of the Klein-Gordon equation in $\mathbf{R}^{n+1}$, Comm. Pure Appl. Math. 46 137-144 (1993)

[37] Kuksin, S.B.: Nearly integrable infinite-dimensional Hamiltonian systems, Lecture Notes in Math. 1556, Springer Verlag, Berlin-Heidelberg-New York, 1993

[38] Lamb, H.: On a peculiarity of the wave-system due to the free vibrations of a nucleus in an extended medium, Proc. London Math. Soc. 32, 208-211 (1900)

[39] Lions, P.-L.: The concentration compactness principle in the calculus of variations, I. The locally compact case, Ann. Inst. Henri Poincaré, Analyse nonlinéaire, 1, 109-145 (1984)

[40] MacKay, R.S., Aubry, S.: Proof of existence of breathers for time-reversible or Hamiltonian networks of weakly coupled oscillators, Nonlinearity 7, 1623 1643 (1994)

[41] McLaughlin, D.W., Scott, A.C.: Perturbation analysis of fluxon dynamics, Phys. Rev. A18 1652 (1978)

[42] McLaughlin, D.W., Shatah, J.: Homoclinic orbits for pde's , Proc. Sympos. Appl. Math. 54, 281-299, AMS Providence, RI 1998

[43] Mourre, E.: Absence of singular continuous spectrum for certain self-adjoint operators, Commun. Math. Phys. 78, 391 (1981) 
[44] Nier, F.: The dynamics of some open quantum systems with short-range nonlinearities, preprint 1997

[45] Nirenberg, L.: Topics in Nonlinear Functional Analysis, Courant Institute Lecture Notes, 1974

[46] Pillet, C.-A., Wayne, C.E.: Invariant manifolds for a class of dispersive, Hamiltonian, partial differential equations, J. Diff. Eqns. 141, 310-326 (1997)

[47] Pyke, R.M., Sigal, I.M.: Nonlinear wave equations: Constraints on periods and exponential bounds for periodic solutions, Duke Math. J. 88, (1997)

[48] Perry, P., Sigal, I.M., Simon, B.: Spectral analysis of N-body Schrödinger operators, Ann. Math 114, 519-567 (1981)

[49] Reed, M., Simon, B.: Modern Methods of Mathematical Physics, Volume 1, Functional Analysis, Academic Press 1972

[50] Reed, M., Simon, B.: Modern Methods of Mathematical Physics, Volume 4, Analysis of Operators, Academic Press 1978

[51] Rose, H.A., Weinstein, M.I.: On the bound states of the nonlinear Schrödinger equation with a linear potential, Physica D 30, 207-218 (1988)

[52] Sanders, J.A., Verhulst, F.: Averaging Methods in Nonlinear Dynamical Systems, Applied Mathematical Sciences 59, Springer-Verlag, New York (1985)

[53] Schulman, L.S., Doering, C.R., Gaveau, B.: Linear decay in multilevel quantum systems, J. Phys. A: Gen. 24, 2053-2060 (1991)

[54] Segur, H., Kruskal, M.D.: Nonexistence of small amplitude breather solutions in $\phi^{4}$ theory, Phys. Rev. Lett., 58, 747 (1987)

[55] Shatah, J., Strauss, W.A.: Breathers as homoclinic geometric wave maps, Physica D (1996)

[56] Sigal, I.M.: Nonlinear wave and Schrödinger equations I. Instability of timeperiodic and quasiperiodic solutions, Commun. Math. Phys. 153, 297 (1993)

[57] Sigal, I.M.: General characteristics of nonlinear dynamics, in Spectral and Scattering Theory; Proceedings of the Taniguchi international workshop, ed. M. Ikawa, Marcel Dekker, Inc. New York-Basel-Hong Kong 1994

[58] Sigal, I.M., Soffer, A.: Local decay and velocity bounds for quantum propagation, preprint, Princeton 1988 (ftp: //www.math.rutgers.edu/pub/soffer)

[59] Skibsted, E.: Propagation estimates for N-body Schrödinger operators, Commun. Math. Phys. 142, 67-98 (1991)

[60] Soffer, A., Weinstein, M.I.: Multichannel nonlinear scattering theory for nonintegrable equations I \& II, Commun. Math. Phys. 133 119-146 (1990); J. Diff. Eqns. 98, 376-390 (1992)

[61] Soffer, A., Weinstein, M.I.: Dynamic theory of quantum resonances and perturbation theory of embedded eigenvalues, in Proceedings of Conference on Partial Differential Equations and Applications, University of Toronto, June, 1995, CRM Lecture Notes, Eds. P. Greiner, V. Ivrii, L. Seco and C. Sulem

[62] Soffer, A., Weinstein, M.I.: Time dependent resonance theory, to appear in Geometric and Functional Analysis

[63] Soffer, A., Weinstein, M.I.: The large time behavior of the nonlinear Schrödinger equation; Selection of the ground state and instability of excited states, in preparation

[64] Stein, E.M.: Singular Integrals and Differentiability Properties of Functions, Princeton University Press (1970)

[65] Strauss, W.A.: Nonlinear Wave Equations, CBMS Regional Conference Series in Mathematics \#7 AMS (1985) 
[66] Stuart, D.M.: Minimal periods for solutions of some classical field equations, SIAM J. Math. Anal. 27, 1095-1101 (1996)

[67] Vanderbauwhede, A., Iooss G.: Center manifold theory in infinite dimensions, Dynamics Reported 2, (1990)

[68] Wayne, C.E.: Periodic and quasi-periodic solutions of nonlinear wave equations via KAM theory, Commun. Math. Phys. 127, 479 (1990)

[69] Weinstein, A.: Periodic nonlinear waves on a half-line Commun. Math. Phys. 99383 (1985); Erratum 107, 177 (1986)

[70] Weinstein, M.I.: Lyapunov stability of ground states of nonlinear dispersive evolution equations, Commun. Pure Appl. Math. 39, 51-68 (1986)

[71] Weisskopf, V., Wigner, E.: Berechnung der natürlichen Linienbreite auf Grund der Diracschen Lichttheorie, Z. Phys. 63, 54-73 (1930)

[72] Yajima, K., $\mathrm{W}^{\mathrm{k}, \mathrm{p}}$ - continuity of wave operators for Schrödinger operators, J. Math. Soc. Japan 47, 551-581 (1995)

[73] Zhang, F., Kivshar, Y.S., Malomed, B.A., Vázquez, L.: Kink capture by a local impurity in the sine Gordon model, Phys. Lett. A159, 318-322f (1991) 